\title{
Presence of solar filament plasma detected in interplanetary coronal mass ejections by in situ spacecraft
}

\author{
Rahul Sharma ${ }^{1, *}$ and Nandita Srivastava ${ }^{2}$ \\ 1 3, Indra Nagar, North Sunderwas, Udaipur 313001, India \\ *corresponding author: e-mail: sharmarahul20@gmail.com \\ 2 Udaipur Solar Observatory, Physical Research Laboratory, Badi Road, Udaipur 313001, India \\ Received 29 February 2012 / Accepted 11 July 2012
}

\section{ABSTRACT}

\begin{abstract}
Aims: To identify the solar filament plasma at $1 \mathrm{AU}$ by using in situ spacecraft data.
Methods: We used magnetic, plasma and compositional parameters to identify the presence of filamentary material within and outside magnetic clouds.

Results: We report two cases of observed filament plasma embedded in interplanetary coronal mass ejections (ICMEs) related to a flare-associated eruptive filament and a quiescent filament eruption at different phases of solar cycle by using magnetic, plasma, and compositional parameters.

Conclusions: Analysis of in situ multi-spacecraft observations of ICME structures and substructures confirms the presence of solar filament material.
\end{abstract}

Key words. interplanetary coronal mass ejection (ICME) - filaments - plasma - solar wind

\section{Introduction}

Coronal mass ejections (CMEs) are explosive events that cause expulsion of huge amount of solar plasma $\left(10^{15}-10^{16} \mathrm{~g}\right)$ in heliosphere (Howard et al. 1982; Hundhausen 1988), resulting into impulsive streams with velocities greater than $600 \mathrm{~km} \mathrm{~s}^{-1}$. Most of these streams are associated with the interplanetary component of CMEs and are termed as interplanetary coronal mass ejections or ICMEs. As seen in coronograph images, the three part structure of ICMEs, a bright front, dark cavity and a bright core (Hundhausen 1988; Alexander et al. 2006; Hudson et al. 2006), is maintained at 1 AU and is believed to be the result of plasma pileup, magnetic flux rope and ejected cold plasma respectively (Klein \& Burlaga 1982; Crooker \& Horbury 2006; Forsyth et al. 2006; Schwenn et al. 2006). Subsequently, the relation between disappearing filaments and ICMEs is well established (Burlaga et al. 1982; Wilson \& Hildner 1984, 1986; Marubashi 1986; Bothmer \& Schwenn 1994). To identify the physical signatures of filament plasma in an ICME, Burlaga et al. (1998) analyzed 6 January 1997, solar disk CME event and found possible relation between magnetic cloud and associated eruptive filament through plasma and magnetic properties. Further, Gopalswamy et al. (1998) identified filament plasma as a pressure pulse in an ICME resulting from 7 February 1997 CME. Yao et al. (2010) used velocity distribution functions from magnetic and plasma features and identified cold dense material in Helios data. Also, Lepri \& Zurbuchen (2010) used data from SWICS instrument onboard the ACE satellite to survey cold density enhancements in ICMEs and found filament imprints as cold material with low charge states.

In the work presented here, we detected solar filament plasma in two ICMEs observed at different phases of the solar cycle, using magnetic, plasma and compositional signatures.
Out of the two events investigated, one is an active region filament which erupted in association with a M3.9/2N flare, while the other is a quiescent eruptive filament.

\section{Signatures of solar filament plasma in ICME}

\subsection{Magnetic and plasma signatures}

To investigate the filament ejecta at 1 AU, Burlaga et al. (1998) used magnetic and plasma signatures which included high electron and proton densities in filament plasma region enriched with $\mathrm{He}^{++}$ions and the presence of singly ionized helium $\left(\mathrm{He}^{+}\right)$. They also reported depressions in thermal velocities (defined as $V_{\text {th }}=\sqrt{2 k T / m}$, where $k$ is the Boltzmann constant, $T$ is the temperature and $m$ is the mass), for $\mathrm{He}^{++}$ion and in ratio of $V_{\mathrm{th}\left(\mathrm{He}^{++}\right)} / V_{\mathrm{th}\left(\mathrm{H}^{+}\right)}$, Gopalswamy et al. (1998) observed a high density pressure pulse with drop in thermal velocity $\left(V_{\text {th }} \leq 20 \mathrm{~km} \mathrm{~s}^{-1}\right)$ following 9-10 February 1997 magnetic cloud associated with a filament eruption. Yao et al. (2010) used three-dimensional thermal velocity distribution functions to identify filament material in three reported events as small and isotropic contours on magnetic field-thermal velocity phase space. Another important signature to identify the filamentary material is the decrease in RMS deviations of bulk velocity $(V)$ and magnetic field strength $(B)$, which were first studied by Pudovkin et al. (1979) and were later reported by Zwickl et al. (1983) in association with the properties of driver gas in an ICME. In this paper, we also computed $\delta V_{\text {rms }}$ (variations in RMS values for proton velocity) at an instance " $i$ " using:

$$
\delta\left(V_{\mathrm{rms}}\right)_{i}=\left(V_{\mathrm{rms}}\right)_{i}-\left(V_{\mathrm{rms}}\right)_{\text {mean }}
$$


where $\left(V_{\text {rms }}\right)_{i}=\sqrt{V_{i}^{2} / \rho_{i}}$, while $\left(V_{\text {rms }}\right)_{\text {mean }}$ is the mean of RMS values for data interval. The filament plasma regions in interplanetary medium are often associated with pressure balanced structures (PBS) (Ferraro \& Plumpton 1966) described as the regions where the sum of magnetic and thermal (proton + electron) pressures remains constant. The existence of such microscale structures $(\leq 1 \mathrm{~h})$ in solar wind was demonstrated by Burlaga (1968). He studied the anti-correlation between magnetic pressure and thermal pressure and showed the equilibrium conditions on microscale $(\leq 1 \mathrm{~h})$ and dynamical hydromagnetic processes on mesoscale ( $\approx 2$ days) (Burlaga \& Ogilvie 1970). Mathematically, an equilibrium with local plasma convected outward with solar wind can be represented as

$$
\frac{B^{2}}{8 \pi}+N_{\mathrm{p}} k T_{\mathrm{p}}+N_{\mathrm{e}} k T_{\mathrm{e}}=\mathrm{C}, \quad(\text { Burlaga 1995) }
$$

where $B$ is the magnitude of the magnetic field, $N_{\mathrm{p}}, N_{\mathrm{e}}, T_{\mathrm{p}}$ and $T_{\mathrm{e}}$ are the respective electron and proton densities and temperatures while $\mathrm{C}$ is an equilibrium constant. For $N_{\mathrm{p}} \cong N$, a linear relation between $B^{2}$ and density across a PBS can be given as $B^{2}=-S N+a$, where $S=8 \pi k\left(T_{\mathrm{p}}+T_{\mathrm{e}}\right)$ and $a=8 \pi \mathrm{C}$ (Burlaga 1995), indicated by a negative slope for a plot between $B^{2}$ versus $N$.

\subsection{Compositional signatures}

The compositional signatures for filamentary plasma have been reported in early works (Bame et al. 1979; Schwenn et al. 1980; Zwickl et al. 1983) which included the density enhancements in helium and other heavy elements in ICMEs. The presence of low charge state carbon $\left(\mathrm{C}^{2+}\right)$, oxygen $\left(\mathrm{O}^{2+}\right)$ and iron $\left(\mathrm{Fe}^{4+}\right)$ along with singly ionized helium (Schwenn et al. 1980) suggests that the plasma must have originated at a lower temperature in solar corona. The event reported by Burlaga et al. (1998), accompanying an observation of disappearing filament and also the one by Skoug et al. (1999), observed that enhanced $\mathrm{He}^{+} / \mathrm{He}^{++}$ratio indicated the presence of prominence material. Lepri \& Zurbuchen (2010) reported observations of low charge state cold plasma by employing velocity and relative charge state abundance criteria for $\mathrm{C}, \mathrm{O}$ and $\mathrm{Fe}$ ions. It is further possible to derive information about the local environment where the plasma might have originated by using freeze-in principle (Hundhausen 1972; Owocki \& Scudder 1983). The charge state ratios measured in interplanetary medium can be converted to freeze-in temperature (Geiss et al. 1995), which is an indicator of coronal temperature at the site of freeze-in process. For a species $\chi$, the conservation equation at an ionization stage " $i$ " can be written as

$$
\nabla\left(n_{i} u_{i}\right)=n_{\mathrm{e}}\left[n_{i-1} C_{i-1}-n_{i}\left(C_{i}+R_{i}\right)+n_{i+1} R_{i+1}\right],
$$

(Hundhausen 1972)

where $C_{i}$ and $R_{i}$ are the electron-temperature-dependent ionization and recombination rate coefficients of $\chi^{i+}$ while $n_{\mathrm{e}}$ and $n_{i}$ are electron and ion densities. The density ratio of adjacent charge state obeys $n_{i+1} / n_{i}=C_{i}\left(T_{\mathrm{e}}\right) / R_{i+1}\left(T_{\mathrm{e}}\right)$, where $T_{\mathrm{e}}$ is the electron temperature. Complete numerical solution to the above expression can be obtained using ionization and recombination rate coefficients given by Shull \& van Steenberg (1982).

\section{Observations}

We have selected two eruptive filaments observed on 18 November 2003 and 1 August 2010, which were associated with CMEs. Their in situ properties were determined using ACE and Wind satellites located at L1 point in the ecliptic plane. We obtained magnetic field data from the Wind/MFI (Lepping et al. 1995) and the plasma data from Wind/3DPlasma Analyzer, Wind/SWE (Ogilvie et al. 1995) instrument. ACE data were acquired by the MAG (magnetic field, Smith et al. 1998), SWEPAM (plasma, McComas et al. 1998) and SWICS (ionic composition, Gloeckler et al. 1998), while $\mathrm{He}^{+}$ data were obtained by CELIAS/STOF (Hovestadt et al. 1995) onboard SOHO satellite. The time resolution ranged from 1 minute to 1 hour for different datasets.

\subsection{November 2003 event}

Magnetic cloud arriving on 20 November 2003 has been associated with a halo CME observed on 18 November at 08:50 UT from active region AR10501 (Gopalswamy et al. 2005). The CME was associated with GOES M3.9/2 N flare event located at N00E18 and is shown in Figure 1. Udaipur Solar Observatory observed the filament eruption in $\mathrm{H} \alpha$ starting at 07:27 UT until 08:14 UT. The eruption was also observed at 08:24 UT by Extreme-ultraviolet Imaging Telescope (EIT) of SOHO and associated CME in Large Angle and Spectrometric COronagraph (LASCO) (Brueckner et al. 1995) images. It was a fast CME propagating with linear velocity $\approx 1660 \mathrm{~km} \mathrm{~s}^{-1}$ recorded in SOHO/LASCO CME catalog (http//:www.cdaw.gsfc.nasa. gov/CME_list), observed in LASCO field of view. The source region of this CME has been extensively studied by Srivastava et al. (2009), Chandra et al. (2010), Chandra et al. (2011) and Schmieder et al. (2011). The associated ICME has been detected close to the Earth by enhancement of density measured by the scintillation method using the Ooty radiograph (Kumar et al. 2011). The CME dynamics is studied by Yurchyshyn et al. (2005), Gopalswamy et al. (2005), Wang et al. (2006). Möstl et al. (2008) reconstructed the magnetic flux rope structure embedded in the associated magnetic cloud by using Grad-Shafranov reconstruction method (Hu \& Sonnerup 2002).

\subsubsection{In situ ICME observations}

The magnetic and plasma properties shown in Figures 2 and 3 respectively correspond to a magnetic cloud which originated on 18 November with a flare-associated filament eruption. In situ observations were taken during the interval from 19-22 November 2003 from Wind spacecraft. The three part structure of the CME as seen in LASCO/C2 observations (10:26 UT on 18 November) arrived at 1 AU with a forward shock indicated in both figures with a red line, followed by sheath and the cloud region whose front and rear boundaries are marked by blue lines. Wind detected a shock at 08:06 UT on 20 November with abrupt increase in magnetic and plasma parameters. Magnitude of magnetic field increased from 9 to $16 \mathrm{nT}$ with increase in bulk velocity from 444 to $546 \mathrm{~km} \mathrm{~s}^{-1}$. Simultaneously, proton density rose from 10 to $14 \mathrm{n} / \mathrm{cc}$ while temperature jumped from 0.5 to $2.4 \times 10^{5} \mathrm{~K}$ (Fig. 3), at the time of shock. The observed proton temperature is compared with $0.5 T_{\exp }$ (half-magnitude of computed expected temperature), computed from

$$
\left(0.031 V_{\mathrm{sw}}-5.1\right)^{2} \times 10^{3}, \quad V_{\mathrm{sw}}<500 \mathrm{~km} \mathrm{~s}^{-1}
$$

$$
\left(0.51 V_{\mathrm{sw}}-142\right)^{2} \times 10^{3}, \quad V_{\mathrm{sw}} \geq 500 \mathrm{~km} \mathrm{~s}^{-1}
$$



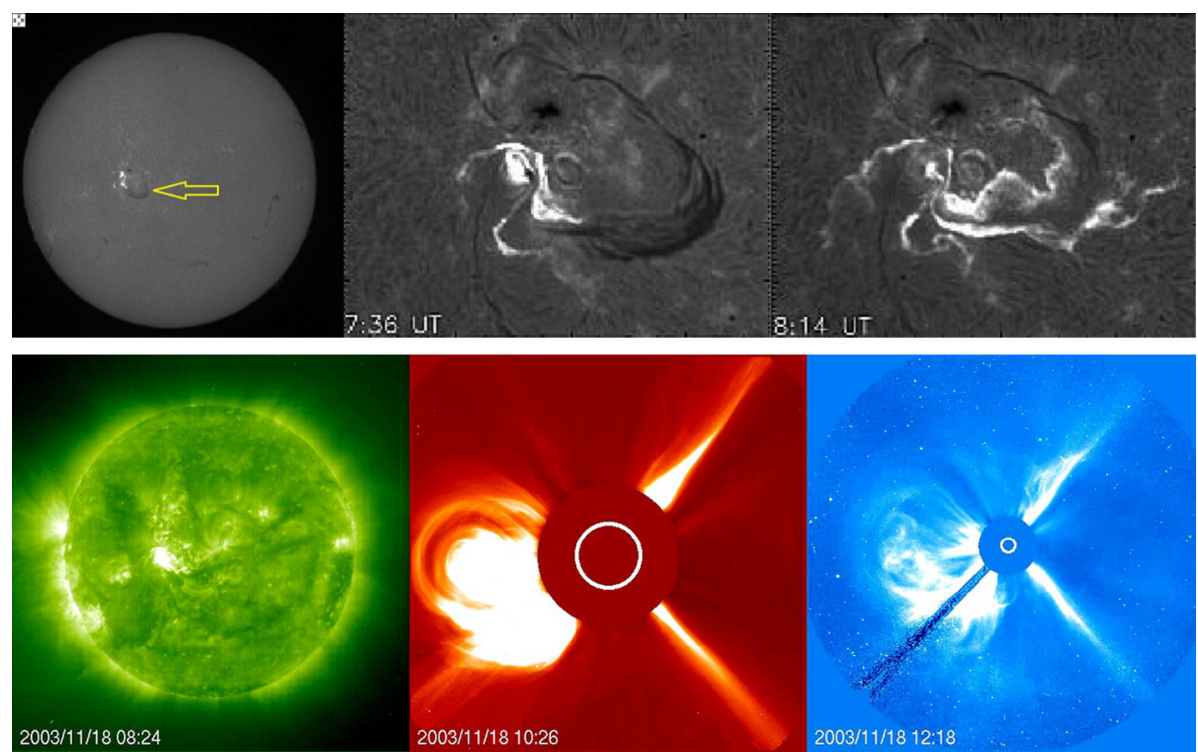

Fig. 1. Top panel: (left to right) $\mathrm{H} \alpha$ filtergram from the Kanzelhöhe Solar Observatory showing eruptive filament (indicated by arrow), solar filament eruption at 07:36 UT with a flare and at 08:14 UT from Udaipur Solar Observatory on 18 November 2003. Bottom panel: Temporal evolution of associated CME in SOHO EIT, LASCO C2 and C3 field of view on 18 November 2003.

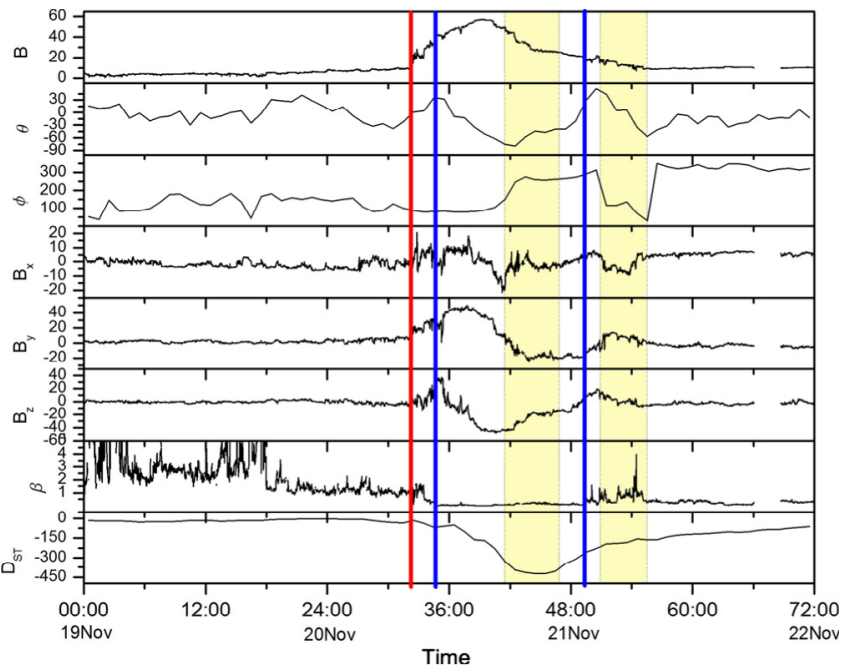

Fig. 2. A plot of interplanetary magnetic field parameters from 19 to 22 November 2003. From top to bottom are plotted the magnetic field strength $(B)$, the elevation $(\theta)$ and azimuth $(\phi)$ of the magnetic field direction, three-dimensional magnetic field components $\left(B_{x}, B_{y}\right.$ $B_{z}$ ), plasma beta obtained from Wind spacecraft and 1-h averaged Dst index. Red line indicates the shock while the region between blue lines is magnetic cloud. Time is plotted in hours starting from 19 November, 00:00 h.

(Lopez \& Freeman 1986; Lopez 1987), where $V_{\text {sw }}$ is the solar wind bulk velocity. These were checked as a signature of ICMEs and were found consistent with previously reported cases (Gosling et al. 1973; Richardson \& Cane 1995). Sheath region embedded in between shock and cloud structure can be identified by compressed magnetic field and plasma parameters with high proton temperature. The magnetic cloud arriving at 10:43 UT on 20 November is clearly distinguished by its relatively high magnetic field strength $(B)$, smooth rotation of elevation angle $(\theta)$ from north to south and low electron and proton temperatures (Fig. 2). The beginning of the cloud is marked by drop in plasma beta below unity $(\beta<1)$ showing the dominance of magnetic pressure in the region.

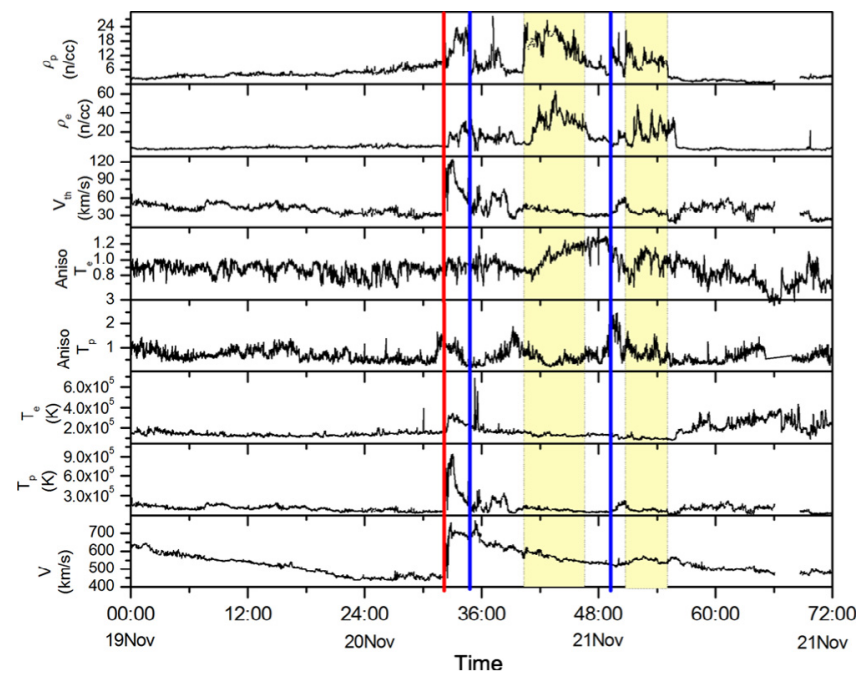

Fig. 3. Measurements of proton density $\left(\rho_{\mathrm{p}}\right)$, electron density $\left(\rho_{\mathrm{e}}\right)$, proton thermal velocity $\left(V_{\mathrm{th}}\right)$, electron temperature anisotropy, proton temperature anisotropy, electron temperature $\left(T_{\mathrm{e}}\right)$, proton temperature $\left(T_{\mathrm{p}}\right)$ and solar wind bulk velocity $(V)$ obtained from Wind spacecraft. Red line indicates the shock while the region between blue lines is magnetic cloud. The yellow-shaded region is filament plasma, indicated by high density (proton and electron) and low temperatures.

With the magnetic cloud arrival, the magnetic field strength rises from 37 to a peak value of $58 \mathrm{nT}$, along with a drop in temperature from $2.6 \times 10^{5} \mathrm{~K}$ to $1.3 \times 10^{5} \mathrm{~K}$. The magnetic cloud resulted in a large geomagnetic storm with Dst $\cong-422 \mathrm{nT}$.

The bulk velocity drops from 688 to $538 \mathrm{~km} \mathrm{~s}^{-1}$ (Fig. 3) during the cloud interval, indicating an expansion in cloud with expansion velocity (Zurbuchen \& Richardson 2006) $V_{\text {exp }}=75 \mathrm{~km} \mathrm{~s}^{-1}$ and an average transverse velocity of $\approx 613 \mathrm{~km} \mathrm{~s}^{-1}$. The presence of flux rope can also be inferred from the bipolar $B_{y}$ and $B_{z}$ components of the magnetic field. The $B_{x}$ component points from the spacecraft toward the Sun while $B_{y}$ points in the ecliptic plane normal to $B_{x}$. The $B_{z}$ 
component is normal to the ecliptic plane toward the north pole. In this case, the $B_{y}$ component varies from $+45 \mathrm{nT}$ (at 13:30 UT) to $-30 \mathrm{nT}$ (at 20:00 UT) on 20 November. Similarly, $B_{z}$ component varies from $+37 \mathrm{nT}$ (at 11:15 UT) to $-47 \mathrm{nT}$ (at 16:00 UT) on 20 November. End of cloud structure is determined by increase in magnitude of $\beta$ over unity at 01:17 UT on 21 November 2003.

\subsubsection{Identification of filament plasma}

\subsubsection{Magnetic and plasma signatures}

Wind detected high proton densities with low temperatures indicating fragmented filamentary material both within and outside the magnetic cloud (shown as yellow-shaded regions in Fig. 3). Inside the cloud region, the filament plasma arrives at Wind on 20 November with sudden rise in proton density from 9 to $22 \mathrm{n} / \mathrm{cc}$. The structure with peak proton density of $26 \mathrm{n} / \mathrm{cc}$ passed the spacecraft, lying over the bipolar $B_{y}$ and $B_{z}$ magnetic field components. This feature is consistent with solar disk observations where the filament is seen to be lying along the inversion line between opposite field polarities. Also enhancements in electron densities were observed in the region where the peak density is about $59 \mathrm{n} / \mathrm{cc}$. Proton thermal velocities show depressions in the filament plasma region with a value of $\approx 38 \mathrm{~km} \mathrm{~s}^{-1}$. The trailing fragment of filament plasma arrives in the interval from 03:12 UT to 06:58 UT on 21 November, following the magnetic cloud. Here, proton densities attain a maximum value of $17 \mathrm{n} / \mathrm{cc}$ with temperature of $\approx 8.0 \times 10^{4} \mathrm{~K}$. Similar trend is observed with electron densities which rise to $44 \mathrm{n} / \mathrm{cc}$ and temperature of $1.0 \times 10^{5} \mathrm{~K}$ in the region. The temperature anisotropy $\left(T_{\perp} / T_{\|}\right)$observations (Bame et al. 1975; Marsch et al. 1982; Feldman et al. 1996; Neugebauer et al. 2001) are supposed to be caused by scattering due to Alfven-cyclotron fluctuations (Marsch \& Tu 2001; Tu \& Marsch 2002; Marsch et al. 2004). In this analysis, the proton temperature anisotropies were found higher than unity $\left(T_{\perp} / T_{\|}>1\right)$ throughout the magnetic cloud and filament regions, while electron temperature anisotropy magnitudes were lower than unity in magnetic cloud region but elevate over unity in filament region during the intervals 18:22 UT on 20 November to $02: 00$ UT on 21 November and 04:14 UT to 06:07 UT on 21 November.

The thermal velocity features of plasma components were computed from the data obtained by Wind and ACE spacecraft. Depressions in parallel component of thermal velocity (Fig. 4) of proton (SWEPAM/ACE) are observed from 14:06 UT to 15:26 UT in magnetic cloud region with a minimum value of about $25 \mathrm{~km} \mathrm{~s}^{-1}$. Similar trend is observed in trailing plasma region from 02:48 UT to 03:42 UT on 21 November with an average magnitude of $\approx 34 \mathrm{~km} \mathrm{~s}^{-1}$. The perpendicular component of proton thermal velocity (SWEPAM/ACE) has a lower magnitude as compared to parallel component of thermal velocity $\left(V_{\text {th } \perp}<V_{\text {th } \|}\right)$, except during the interval 01:22-02:00 UT on 21 November. The perpendicular proton thermal velocity shows depressions from 14:00 UT to 15:00 UT in cloud region with an observed minimum of $24 \mathrm{~km} \mathrm{~s}^{-1}$ at 14:32 UT. During the trailing plasma region, the thermal velocity shows lower magnitude from 03:00 UT to 07:49 UT on 21 November while having a minimum value $\approx 17 \mathrm{~km} \mathrm{~s}^{-1}$. Thermal velocity for alpha particle (Fig. 4) is computed from data obtained by 3DP/Wind during the interval 19-22 November 2003. It shows depressions from 14:51 UT (on 20 November) to 00:55 UT (on 21 November) with a minimum $\approx 9 \mathrm{~km} \mathrm{~s}^{-1}$ (at 15:09 UT on 20 November). This trend is also observed from 03:00 UT to

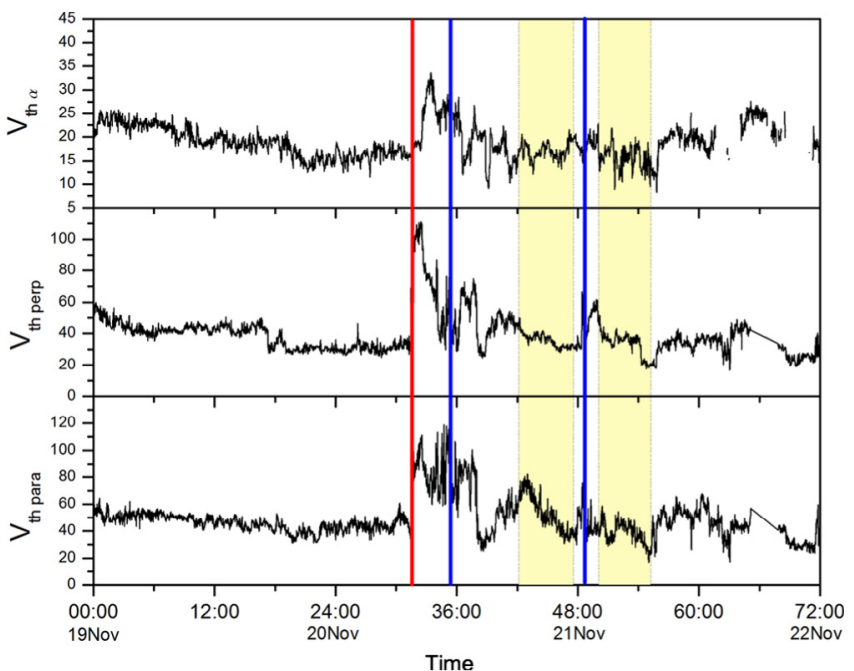

Fig. 4. Measurements of $\mathrm{He}^{2+}$ thermal velocity $\left(\mathrm{km} \mathrm{s}^{-1}\right)$ from $3 \mathrm{DP} /$ Wind data. Perpendicular $\left(V_{\text {thperp }}\right)$ and parallel ( $\left.V_{\text {thpara }}\right)$ components of proton thermal velocity $\left(\mathrm{km} \mathrm{s}^{-1}\right)$ from data by SWEPAM/ACE during the interval 19-22 November 2003. Shaded portion is identified filament plasma.

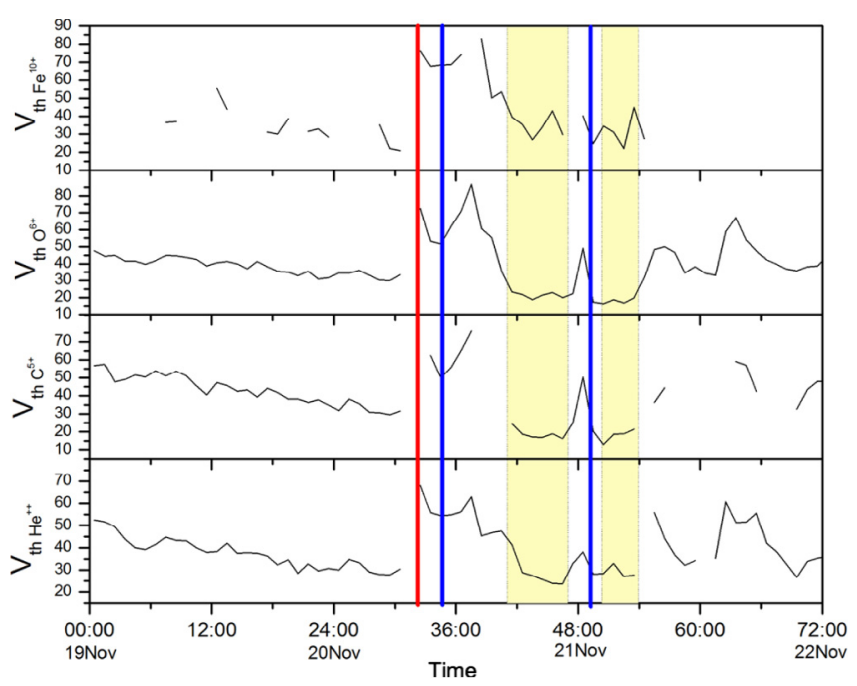

Fig. 5. Plot showing measurements of (top to bottom); iron $\left(\mathrm{Fe}^{10+}\right)$, oxygen $\left(\mathrm{O}^{7+}\right)$, carbon $\left(\mathrm{C}^{5+}\right)$ and helium $\left(\mathrm{He}^{2++}\right)$ thermal velocities from SWICS/ACE data. All velocities are expressed in $\mathrm{km} \mathrm{s}^{-1}$.

07:00 UT on 21 November in trailing plasma region where it shows a minimum $\approx 8 \mathrm{~km} \mathrm{~s}^{-1}$.

Thermal velocities (Fig. 5) of helium $\left(\mathrm{He}^{2+}\right)$, carbon $\left(\mathrm{C}^{5+}\right)$, oxygen $\left(\mathrm{O}^{7+}\right)$ and iron $\left(\mathrm{Fe}^{10+}\right)$ ions are taken from SWICS/ACE data from 19 to 22 November 2003. Helium ion $\left(\mathrm{He}^{2++}\right)$ thermal velocity shows depressions during the interval 18:30 UT (on 20 November) to 00:30 UT (on 21 November) with a minimum $24 \mathrm{~km} \mathrm{~s}^{-1}$ and also from 01:30 UT to 04:30 UT on 21 November as a part of trailing filament plasma region with a minimum magnitude of $27 \mathrm{~km} \mathrm{~s}^{-1}$. Similar trend is observed in carbon $\left(\mathrm{C}^{5+}\right)$ thermal velocity which lowers during the interval from 18:30 UT to 22:30 UT on 20 November in magnetic cloud structure with a minimum $16 \mathrm{~km} \mathrm{~s}^{-1}$. During the trailing plasma region, its magnitude shows depressions from 02:30 UT to $04: 30$ UT on 21 November with a minimum of $\approx 12 \mathrm{~km} \mathrm{~s}^{-1}$. Oxygen $\left(\mathrm{O}^{7+}\right)$ thermal velocity drops from 19:30 UT to 


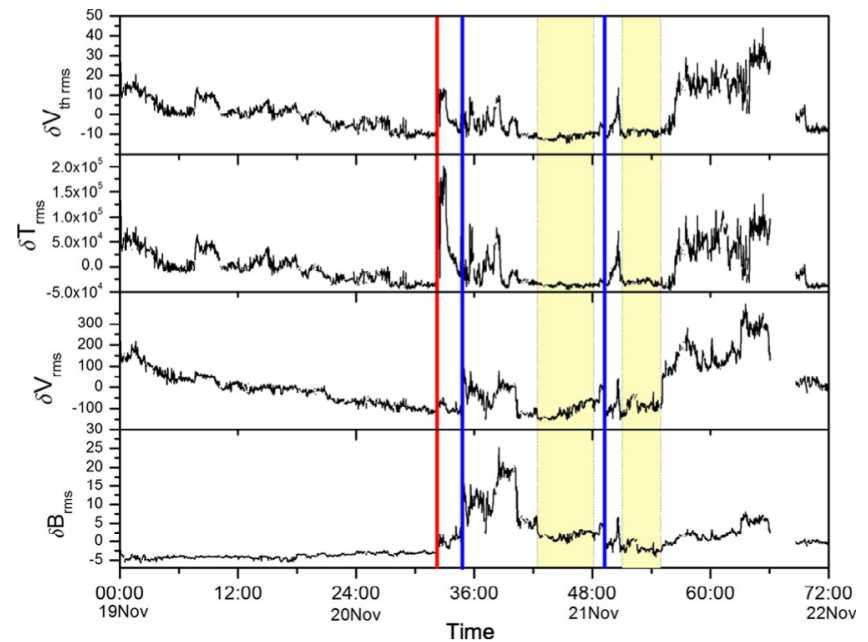

Fig. 6. RMS deviations of plasma and magnetic parameters from data obtained from Wind in November 2003, event.

22:30 UT on 20 November with an average value of $\approx 18 \mathrm{~km} \mathrm{~s}^{-1}$ in magnetic cloud while a minimum of $\approx 16 \mathrm{~km} \mathrm{~s}^{-1}$ in the trailing region is believed to be associated with filament plasma. Iron $\left(\mathrm{Fe}^{10+}\right)$ thermal velocities are available with data gaps during observation period. From the data available, it is found that the magnitude of iron thermal velocity is $\approx 26 \mathrm{~km} \mathrm{~s}^{-1}$ at 19:30 UT on 20 November within the magnetic cloud region while $\approx 22 \mathrm{~km} \mathrm{~s}^{-1}$ at $04: 30$ UT on 22 November as a part of trailing plasma region.

Figure 6 shows the deviations in RMS values for thermal velocity, temperature, bulk velocity and magnetic field. Thermal velocity deviations acquire nearly constant negative values at 17:00 UT on 20 November, lasting until 00:45 UT on 21 November. Depressions are also seen at 03:00 UT to 08:00 UT on 21 November. Similar trend is observed in RMS deviations of temperature which decrease at 17:00 UT on 20 November to 01:00 UT on 21 November and 03:00 UT to 08:00 UT on 21 November. The bulk velocity also shows a decrease in RMS deviation from 18:30 UT to 20:30 UT on 20 November and from 03:00 UT to 06:10 UT on 21 November, while magnetic field RMS values decrease and remain steady from 18:30 UT on 20 November to $00: 45$ UT on 21 November followed by depressions on $04: 30$ UT to $07: 10$ UT on 21 November indicating the presence of cold filament plasma.

Data from Wind/SWE, Wind/3DP and Wind/MFI instruments were used to compute thermal (electron + proton) and magnetic pressures $\left(B^{2} / 8 \pi\right)$ during the interval 12:00-18:00 UT on 20 November and 02:00-04:00 UT on 21 November (Fig. 7). Microscale structures of the order of few minutes were observed on 20 and 21 November as a part of magnetic cloud and trailing region. On 20 November, two structures were identified during 13:00-13:15 and 16:20-16:40 UT followed by more structures on 21 November at 01:10-01:30 UT and 02:00-02:10 UT.

Total pressure remained constant during the observed PBS with an error of $\leq 7 \%$ resulting due to absence of the contribution by alpha particles (Burlaga et al. 1990). Square of magnitude of magnetic field strength $\left(B^{2}\right)$ is plotted with proton density from $1 \mathrm{~min}$ averaged data and found to have a negative slope during intervals of PBS. Figure 8 shows plot of $B^{2}$ versus proton density for intervals 13:00-13:15 UT on 20 November, with the regression coefficient $(R=-0.93)$.

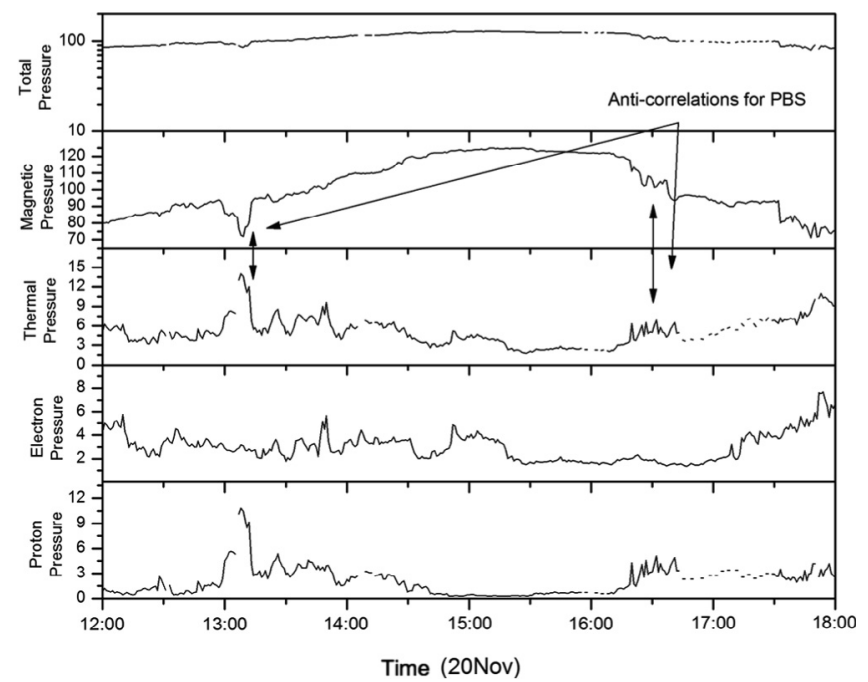

Fig. 7. Plot for computed plasma pressure and magnetic pressure with anti-correlations indicated by arrows during interval 12:00 18:00 UT on 20 November 2003. All parameters are expressed in units of $10^{-10}$ dyn $\mathrm{cm}^{-2}$.

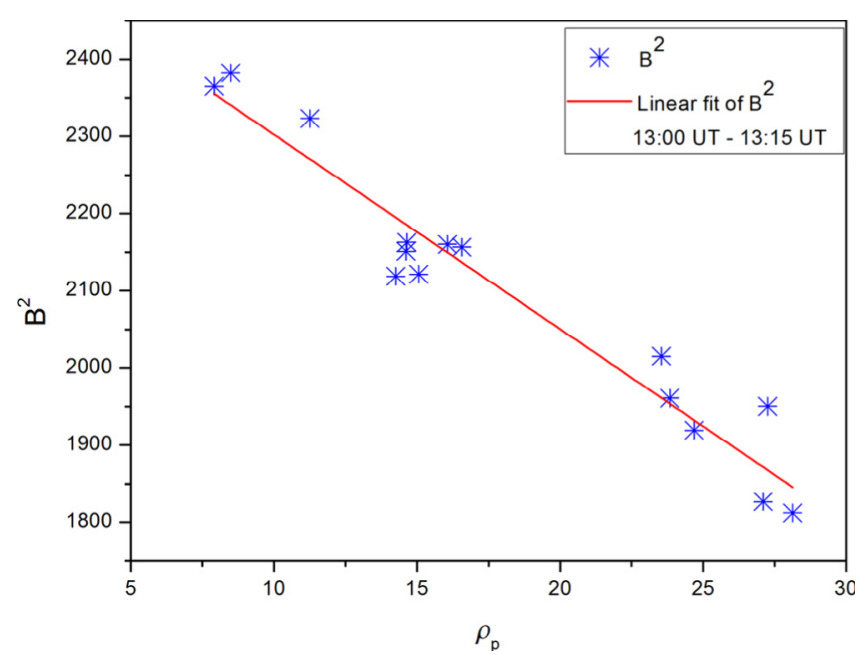

Fig. 8. Plot showing square of magnetic field strength (B) versus proton density $\left(\rho_{\mathrm{p}}\right)$ for time intervals during 20 November 2003.

\subsubsection{Compositional signatures}

Figure 9 shows plots of average carbon, oxygen and iron charge states along with alpha-to-proton ratio obtained from the hourly average data from SWICS instrument onboard ACE spacecraft. The region as identified to be associated with filamentary plasma passed the spacecraft during interval 17:00-22:00 UT on 20 November and 02:30 UT-05:30 UT on 21 November, showing a decrease in carbon and oxygen charge states. Carbon charge state dips to 4.8 as compared to an average 5.5 in ambient plasma while oxygen charge state gets a minimum value of 6.0 within the magnetic cloud region and about 6.2 in the plasma trailing the magnetic cloud on 21 November. The iron charge state remains nearly constant. The region is also associated with increase in proton, electron, ion and helium densities. The solar wind originates from open magnetic field regions (Ogilvie \& Hirshberg 1974) of the corona and has $\mathrm{He}^{2+} / \mathrm{He}^{2+}$ ratio at about 0.05 in fast wind and even lower in slow wind (von Steiger et al. 1995). CMEs associated with filament eruptions originate from closed field regions and can 


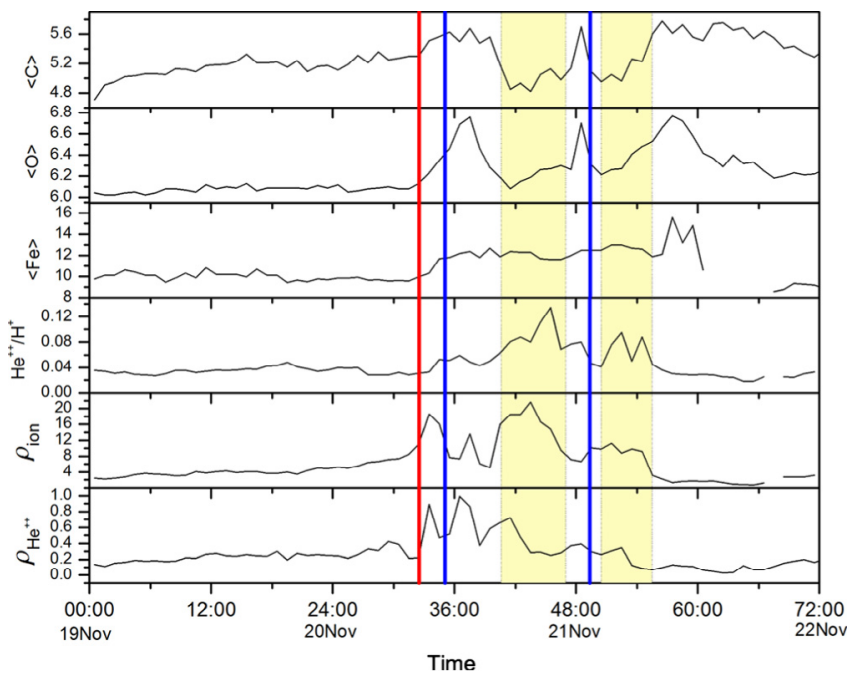

Fig. 9. Compositional signatures of filament plasma from 1-h averaged ACE/SWICS data from 19 to 22 November 2003. Top to bottom: average charge distribution (carbon, oxygen, iron), alpha-toproton ratio, ion density and helium density.

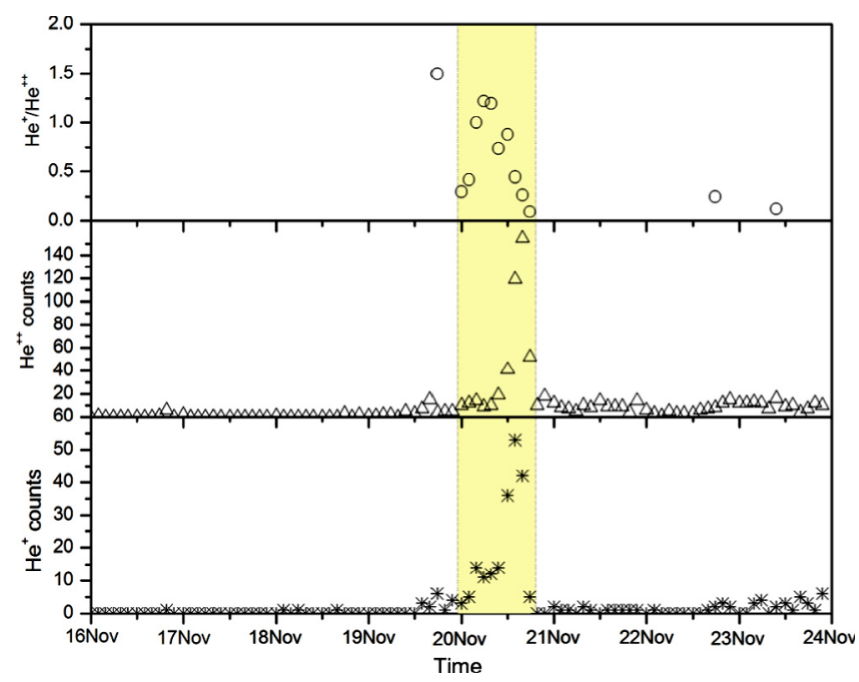

Fig. 10. Helium count rates and ratio from CELIAS/STOF onboard SOHO for 20 November 2003 ICME. Count rates in 100-300 keV energy range are marked as star for $\mathrm{He}^{+}$counts, triangle for $\mathrm{He}^{++}$ counts and circles for the $\mathrm{He}^{+} / \mathrm{He}^{++}$ratio (courtesy: Dr. Martin Hilchenbach).

have up to $30 \%$ enhancement in helium abundances (Galvin 1997). Alpha-to-proton ratio is nearly three times in the filamentary plasma region from 17:00 to 22:00 UT on 20 November in magnetic cloud. The observed elemental charge states $\left(\mathrm{C}^{5+}, \mathrm{O}^{5+}, \mathrm{Fe}^{11+}\right)$ are also two to three times higher as compared to the compositional signatures for prominence plasma $\left(\mathrm{C}^{5+}\right.$, $\mathrm{O}^{2+}, \mathrm{Fe}^{4+}$ ) reported by Lepri \& Zurbuchen (2010), which is possibly due to eruption of filament with flare.

Measurements of $\mathrm{He}^{+}$and $\mathrm{He}^{++}$in November 2003 were made using data from CELIAS/STOF instrument onboard SOHO spacecraft and are shown in Figure 10. The count rates of $\mathrm{He}^{+}$and $\mathrm{He}^{++}$are plotted from 16-24 November 2003. Count rates for $\mathrm{He}^{+}$and $\mathrm{He}^{++}$are significantly higher on 20 November, along with the high count rates of $\mathrm{He}^{+} / \mathrm{He}^{++}$ratio which corresponds to the filamentary material. CELIAS/STOF

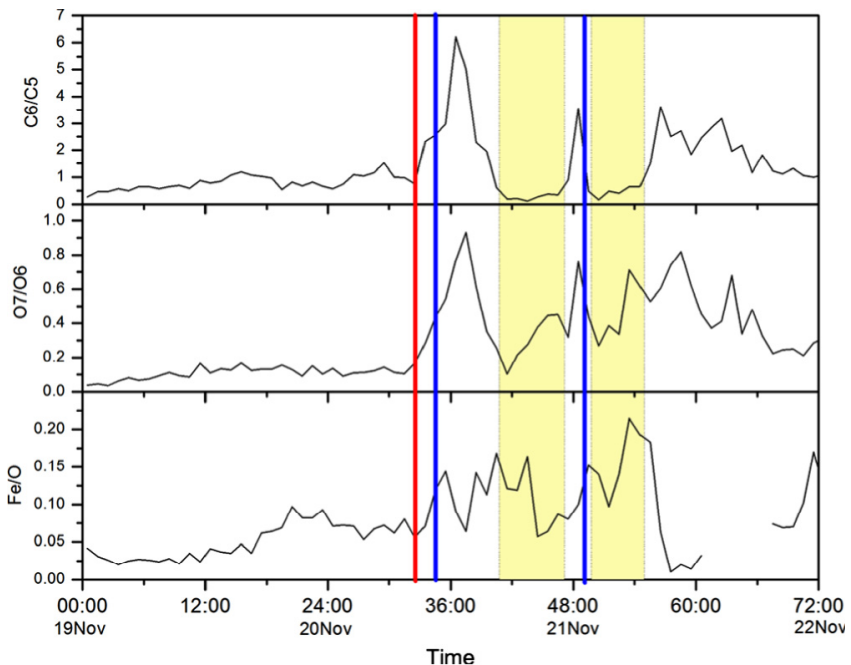

Fig. 11. Charge state ratios of carbon and oxygen ions with $\mathrm{Fe} / \mathrm{O}$ ratio from 19 to 22 November 2003 obtained from SWICS/ACE $-1 \mathrm{~h}$ resolution data.

recorded a maximum 53 counts for $\mathrm{He}^{+}, 155$ counts for $\mathrm{He}^{++}$ and a ratio 1.2 for $\mathrm{He}^{+} / \mathrm{He}^{++}$within magnetic cloud region on 20 November. In combination with other plasma features, these provide an important clue to associate magnetic cloud with prominence plasma.

The variation in charge state ratios of carbon and oxygen ions was also studied from SWICS/ACE 1-h averaged data during the interval 19-22 November 2003. Figure 11 shows the passage of plasma associated with filament, during the interval 16:30-23:30 UT on 20 November and 01:30-05:30 UT on 21 November 2003. In this interval, the $C^{6} / C^{5}$ ratio drops below unity with a minimum value 0.12 inside the cloud and a ratio of 0.16 in trailing plasma structure. Similar trend is observed in oxygen charge state ratios $\left(\mathrm{O}^{7} / \mathrm{O}^{6}\right)$ which acquires a minimum of about 0.10 (at 17:30 UT on 20 November) while 0.26 (at 02:30 UT on 21 November) in trailing region behind the magnetic cloud. The $\mathrm{Fe} / \mathrm{O}$ ratio gets elevated in filament plasma and reaches a maximum of about 0.16 in magnetic cloud and 0.21 in trailing plasma as compared to 0.02 in ambient plasma. The enhancement is around 8 times in cloud region and 10.5 times for plasma trailing the magnetic cloud.

Corresponding freezed-in temperatures for observed charge state ratios were obtained numerically using the relation given by Hundhausen et al. (1968a, 1968b) and ionization and recombination rate coefficients given by Shull \& van Steenberg (1982). For carbon charge state ratio of 0.12 in filamentary plasma region, the computed freezed-in temperature is found to be $5.85 \times 10^{5} \mathrm{~K}$, while for oxygen charge state ratio of 0.10 , the freezed-in temperature is $2.23 \times 10^{5} \mathrm{~K}$.

\subsection{August 2010 event}

Two ICMEs were detected by Wind spacecraft at 04:30 UT (MC1) and 10:32 UT (MC2) on 4 August 2010. These were associated with two filament eruptions observed on 1 August 2010. $\mathrm{H} \alpha$ solar disk observations show a polar crown filament at AR1092 in western hemisphere, stretching from N60W0 to $\mathrm{N} 30 \mathrm{~W} 50$ while the other at N35W0. Both these filaments are marked as northern (NF) and southern (SF) filaments in Figure 12. The northern filament lifted off from solar surface at 09:30 UT and is observed by SDO/AIA (Lemen et al. 2011) 


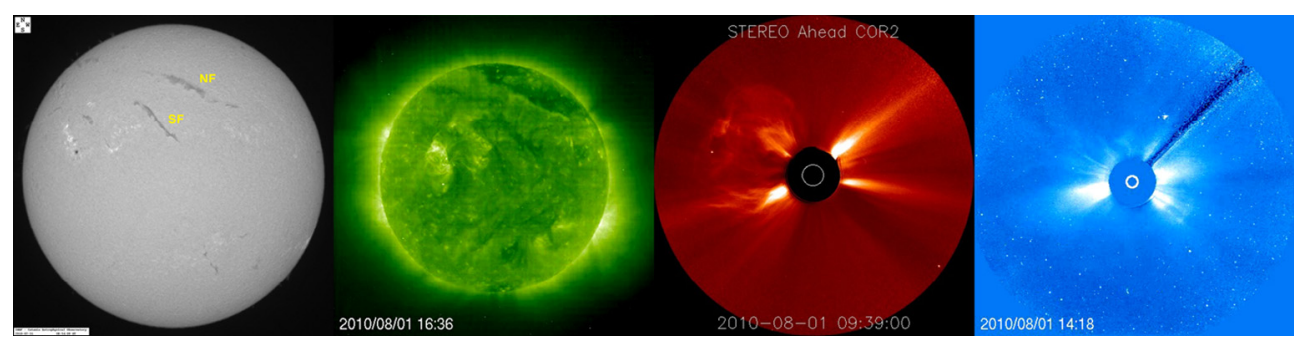

Fig. 12. (Left to right) $\mathrm{H} \alpha$ filtergram from the Kanzelhöhe Solar Observatory showing eruptive filaments (indicated as NF and SF), temporal evolution of associated CME in SOHO-EIT, STEREO-A/COR2, LASCO-C3 on 1 August 2010.

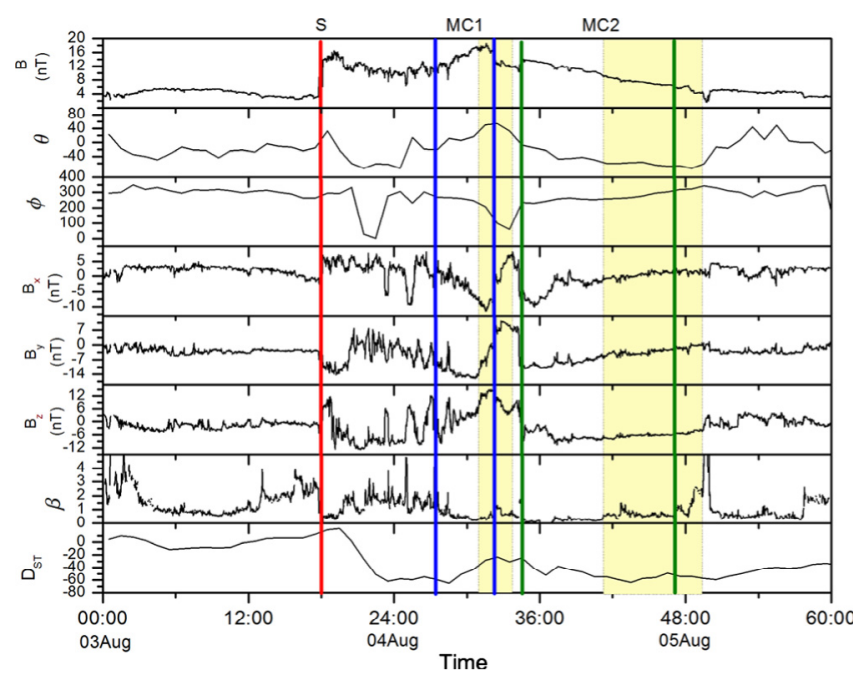

Fig. 13. A plot of magnetic field parameters from 3 to 5 August (12:00 UT), 2010. Top to bottom: the magnetic field strength $(B)$, the elevation $(\theta)$ and azimuth $(\phi)$ of the magnetic field direction, threedimensional magnetic field components $\left(B_{x}, B_{y}, B_{z}\right)$, plasma beta obtain from Wind spacecraft and 1-h averaged Dst index. Red line indicates the shock while the region between blue lines is first magnetic cloud (MC1) followed by second magnetic cloud (MC2) shown between green lines.

while the southern filament erupted at 22:24 UT and was seen in STEREO-A/COR2 (Howard et al. 2008) images. The interplanetary dynamics of associated CMEs have been studied by Harrison et al. (2012) and Temmer et al. (2012).

\subsubsection{In situ ICME observations}

Magnetic and plasma observations taken from Wind spacecraft during 3-5 August 2010 indicate passage of two consecutive magnetic clouds following a shock (S) and sheath structure shown in Figures 13 and 14, identified from magnetic and plasma parameters with one-minute resolution data. Diagnostics of magnetic and plasma features indicate a shock arriving at Wind at 17:47 UT on 3 August. With the shock, magnetic field strength jumped from approx 3 to $8 \mathrm{nT}$ while bulk velocity increased from 414 to $472 \mathrm{~km} \mathrm{~s}^{-1}$. Also, proton density rises from 4 to $8.5 \mathrm{n} / \mathrm{cc}$ with rise in temperature from $1.9 \times 10^{4}$ to $3.4 \times 10^{4} \mathrm{~K}$. The shock related-jump conditions were also observed in proton thermal velocity, electron density, $B_{x}$ and $B_{z}$ components of magnetic field vector. Following the shock is the sheath region, identified by compressions in magnetic and plasma parameters. During this interval, proton temperature increased up to $4.5 \times 10^{5} \mathrm{~K}$ with fluctuating magnetic field strength around $11 \mathrm{nT}$ and the ICME also caused a moderate

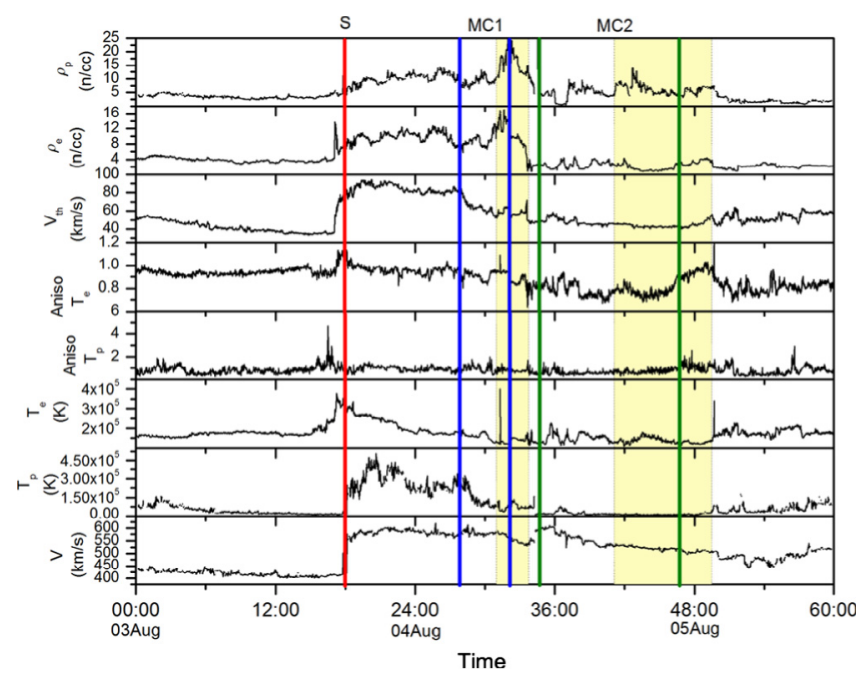

Fig. 14. Measurements of proton density, electron density, proton thermal velocity $\left(V_{\text {th }}\right)$, electron temperature anisotropy, proton temperature anisotropy, electron temperature $\left(T_{\mathrm{e}}\right)$, proton temperature $\left(T_{\mathrm{p}}\right)$ and solar wind bulk velocity $(V)$ obtained from Wind spacecraft for 4 August ICME.

geomagnetic storm (Gonzalez et al. 1994) which reached to a Dst value of $-64 \mathrm{nT}$.

\subsubsection{Identification of filament plasma}

\subsubsection{Magnetic and plasma signatures}

The shock-sheath region is followed by a magnetic cloud (MC1) which resulted from a polar filament eruption on 1 August 2010 as observed by STEREO-A/COR2 (09:54 UT). MC1 passed during the interval from 04:20 to $08: 17$ UT on 4 August. Front boundary of the cloud is marked with a drop in proton temperature from 2.7 to $2.3 \times 10^{5} \mathrm{~K}$ and in plasma $\beta$ from 2.22 to 0.77 . The peak value of magnetic field reached a maximum $18 \mathrm{nT}$ with proton temperature $1.01 \times 10^{5} \mathrm{~K}$ and electron density peaked at about $16 \mathrm{n} / \mathrm{cc}$. The bulk velocity changed from 583 to $576 \mathrm{~km} \mathrm{~s}^{-1}$ during the cloud interval, indicating an expanding cloud structure. The expanding velocity $\left(V_{\exp }\right)$ was found to be $3.5 \mathrm{~km} \mathrm{~s}^{-1}$ with a transverse velocity $\approx 580 \mathrm{~km} \mathrm{~s}^{-1}$. Magnetic field components showed a change from negative to positive values within the cloud. $B_{z}$ changed from -10 (at 03:53 UT on 4 August) to $11 \mathrm{nT}$ (at 08:49 UT on 4 August) with similar changes in $B_{y}$ component which varies from -15 (at 06:24 UT on 4 August) to $11 \mathrm{nT}$ (at 08:49 UT on 4 August). Elevation angle $\theta$ changes from $21.2^{\circ}$ at $03: 30 \mathrm{UT}$ to $53.6^{\circ}$ at $08: 30 \mathrm{UT}$ on 4 August while azimuth angle $\phi$ varies from $270^{\circ}$ at $03: 30$ UT to $98.7^{\circ}$ at $08: 30$ 
UT on 4 August, indicating a west-east orientation in magnetic flux rope configuration. The end of the cloud is marked with increase in magnitude of plasma $\beta$ over unity at 08:17 UT on 4 August.

Second magnetic cloud (MC2) closely followed the first cloud (MC1) and arrived at 10:32 UT on 4 August with rise in magnetic field and a drop in plasma $\beta$ below unity. This cloud is associated with filament eruption (SF) on 1 August (Fig. 12) and was a CME in STEREO-A/COR2 at 22:24 UT. The magnetic field vector rises up to $14 \mathrm{nT}$ in cloud region with average proton and electron temperatures $1.84 \times 10^{4} \mathrm{~K}$ and $1.45 \times 10^{5} \mathrm{~K}$ respectively. The elevation angle varies from $-6.6^{\circ}$ to $-67.7^{\circ}$ while $\phi$ changed from $234.3^{\circ}$ to $321.1^{\circ}$ in cloud interval. The $B_{y}$ component changes from $-11 \mathrm{nT}$ (at 10:33 UT) to $-2 \mathrm{nT}$ (at 22:51 UT) while $B_{z}$ remains negative with very low variance in cloud region, indicating that the spacecraft might have had a glancing encounter with the cloud. Bulk velocity during the cloud interval declines from 592 to $509 \mathrm{~km} \mathrm{~s}^{-1}$ indicating an expanding structure with velocity $\left(V_{\exp }\right)$ approx $41 \mathrm{~km} \mathrm{~s}^{-1}$ and a transverse velocity approx $550 \mathrm{~km} \mathrm{~s}^{-1}$.

Plasma and magnetic observations confirm the presence of filament plasma at the rear of magnetic clouds ( $\mathrm{MC1}$ and MC2). High density and low temperature plasma passes the spacecraft at the rear boundary of $\mathrm{MC1}$ during the interval from 07:11 UT to 09:30 UT on 4 August. This consists of high proton, electron and ion densities with low temperature of the order of $10^{4} \mathrm{~K}$. Peak value of proton density reaches $24 \mathrm{n} / \mathrm{cc}$ with proton temperature of about $7.19 \times 10^{4} \mathrm{~K}$ and electron temperature of about $1.37 \times 10^{5} \mathrm{~K}$ with peak electron density about $17 \mathrm{n} / \mathrm{cc} . B_{y}$ component shows a change from -12 to $12 \mathrm{nT}$ in this region indicating a flux rope configuration. Presence of filamentary plasma over flux rope structure is consistent with the fact that filaments form over the inversion line between opposite magnetic polarities (Martens \& Kuin 1989; Martens \& Zwaan 2001).

Also, regions with high density and low temperature were observed in rear of MC2 during the interval 13:12 UT on 4 August to 01:45 UT on 5 August. This region appears like a diffused plasma section of the filament material encountered by Wind. Peak proton density reaches a value of $7 \mathrm{n} / \mathrm{cc}$ with extremely low temperature $1.6 \times 10^{4}$. Also, low values of electron densities are recorded with peak at $\approx 4 \mathrm{n} / \mathrm{cc}$ and temperature $1.4 \times 10^{5} \mathrm{~K}$. Electron anisotropies remain below unity during entire filament plasma and cloud region in $\mathrm{MC1}$ while proton temperature anisotropies were above unity during interval 04:30-06:30 UT in early part of $\mathrm{MC1}$, but were below unity in filament plasma region. In case of second magnetic cloud (MC2), the electron anisotropies are well below unity but proton anisotropies fluctuated around unity till 12:30 UT. It increased above unity in the rear of MC2 at 23:30 UT indicating presence of Alfven-cyclotron fluctuations (Gary et al. 2006).

The plasma and magnetic signatures for filament plasma identification are conspicuously visible for first magnetic cloud (MC1) but not in second magnetic cloud (MC2). The possible explanation could be the location of spacecraft (Wind and ACE) which made a glancing encounter with one of the flank regions of the MC2. This is also confirmed by the GradShafranov reconstruction of the magnetic cloud by Möstl et al. (2012) and supports the view that the spacecraft location plays a key role in identification of ICME structures and the nature of plasma within (Gopalswamy 2006). In this case, it seems that the spacecraft trajectory encountered the MC1 axis

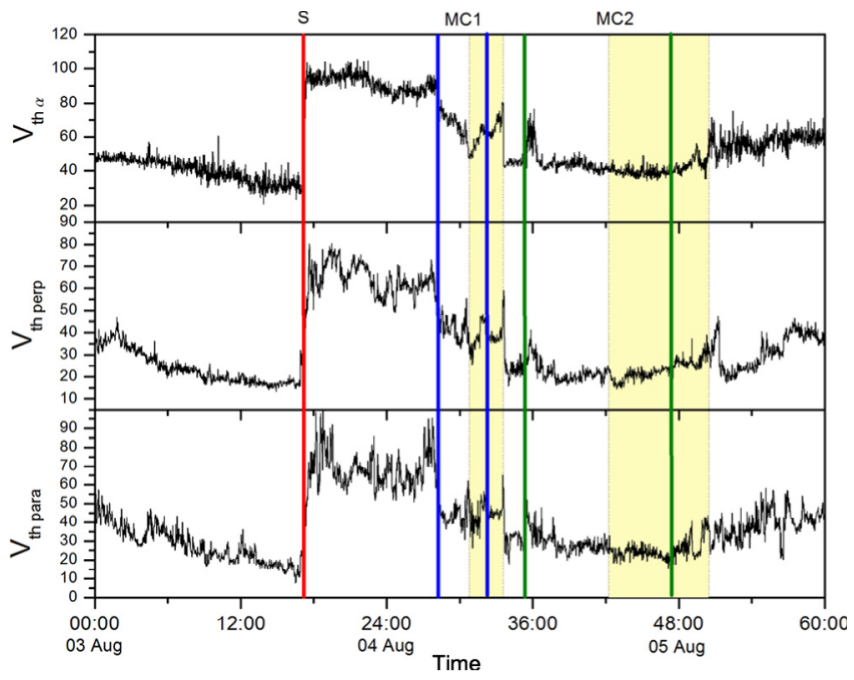

Fig. 15. Measurements of $\mathrm{He}^{++}$thermal velocity $\left(\mathrm{km} \mathrm{s}^{-1}\right)$ from 3DP/Wind data. Perpendicular $\left(V_{\text {thperp }}\right)$ and parallel $\left(V_{\text {thpara }}\right)$ components of proton thermal velocity $\left(\mathrm{km} \mathrm{s}^{-1}\right)$ from data by SWEPAM/ACE during the interval 3-5 (12:00 UT) August 2010. Shaded portion is filament plasma region.

while skimmed one of the flanks of MC2, indicated by low varying magnetic field components and low density plasma; related to filament eruption on 1 August 2010.

Data from SWEPAM/ACE, 3DP/Wind and SWICS/ACE have been used to study the nature of thermal velocities of ionic species during the ICME (MC1 and MC2) interval. The computed thermal velocity component in parallel direction (Fig. 15) shows a decreasing trend starting from 18:00 UT on 4 August until 00:30 UT on 5 August. At the rear boundary of $\mathrm{MC} 1$, the parallel proton thermal velocity drops from 64 to $18 \mathrm{~km} \mathrm{~s}^{-1}$, indicating the presence of cold filament material. In the second magnetic cloud (MC2), its value is lowest and approx $24 \mathrm{~km} \mathrm{~s}^{-1}$. The perpendicular component of proton thermal velocity shows depressions during the interval 13:30 23:45 UT on 4 August and had minimum of about $\approx 16 \mathrm{~km} \mathrm{~s}^{-1}$. The alpha thermal velocity (Fig. 15) computed from data obtained by 3DP/Wind instrument shows a high magnitude, nearly constant thermal velocity during the interval 18:00-23:00 UT on 4 August with an average magnitude $\approx 39 \mathrm{~km} \mathrm{~s}^{-1}$ in MC2. At the rear boundary MC1, the magnitude suddenly drops from $\approx 74$ to $42 \mathrm{~km} \mathrm{~s}^{-1}$ at 09:50 UT on 4 August indicating the presence of low temperature plasma.

SWICS/ACE observations for ionic thermal velocities (Fig. 16) show depressions in helium thermal velocity from 15:00 UT until 23:00 UT on 4 August with a minimum $\approx 11 \mathrm{~km} \mathrm{~s}^{-1}$. There were data gaps in observed carbon thermal velocities but with the available data, it has been observed that the magnitude drops during 10:00 UT (on 4 August)-00:00 UT (on 5 August) with a minimum value $\approx 7 \mathrm{~km} \mathrm{~s}^{-1}$. The oxygen thermal velocity shows low magnitude during 12:00 UT (on 4 August)-00:00 UT (on 5 August) with a minimum observed value of about $\approx 7 \mathrm{~km} \mathrm{~s}^{-1}$. At the rear boundary of $\mathrm{MC1}$, the magnitude of oxygen thermal velocity drops to $\approx 18 \mathrm{~km} \mathrm{~s}^{-1}$. Data for iron thermal velocities are not available during the observation period though a magnitude of $\approx 11 \mathrm{~km} \mathrm{~s}^{-1}$ is observed at rear end of MC1.

Figure 17 shows plots of deviations in temperature, magnetic field with bulk and thermal velocities, calculated from 
R. Sharma and N. Srivastava: Solar filament plasma identification in ICMEs

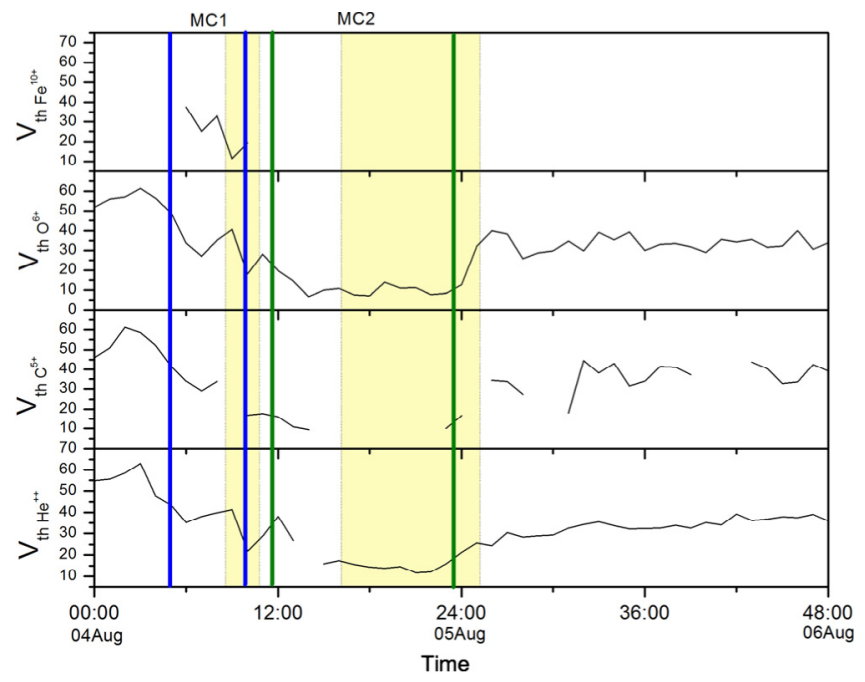

Fig. 16. (top to bottom) Plot showing measurements of; iron $\left(\mathrm{Fe}^{10+}\right)$, oxygen $\left(\mathrm{O}^{7+}\right)$, carbon $\left(\mathrm{C}^{5+}\right)$ and helium $\left(\mathrm{He}^{++}\right)$thermal velocities from SWICS/ACE instruments. All velocities are expressed in $\mathrm{km} \mathrm{s}^{-1}$.

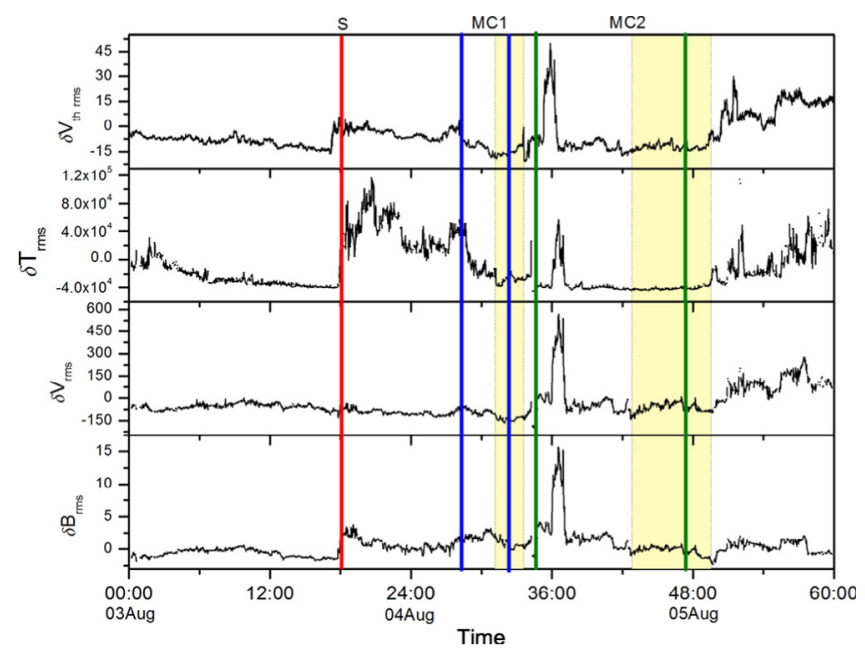

Fig. 17. RMS deviations in (top to bottom) thermal velocity, temperature, bulk velocity, magnetic field from 3 August (00:00) to 5 August (12:00), 2010 ICMEs.

Wind data. Decrease in the RMS deviations of these parameters is taken as signatures of filamentary material along with other plasma and magnetic observations. The depressions indicate the presence of prominence plasma at rear of $\mathrm{MC} 1$ and $\mathrm{MC} 2$ arriving on 4 August at 04:30 UT and 10:32 UT respectively.

RMS deviations in magnetic field reached negative values from 07:05 UT to 09:02 UT on 4 August and from 13:00 UT on 4 August till 01:00 UT on early 5 August. Similar observations were seen in temperature deviations which were low and nearly constant from 13:00 UT (4 August) to 02:00 UT (5 August). Bulk and thermal velocity deviations are also low during same region. These observations along with other magnetic and plasma signatures indicate the presence of cold filamentary plasma.

Figure 18 shows a mesoscale plot of proton, electron, thermal, magnetic and total pressure over an interval of day,

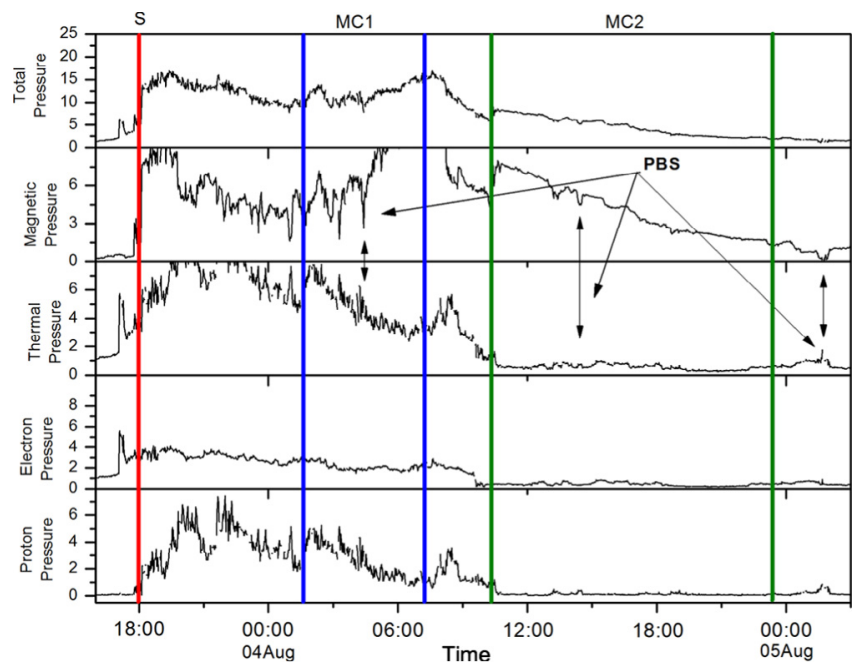

Fig. 18. Plot for computed plasma pressure and magnetic pressure with anti-correlations during interval 15:00 UT on 3 August 2010 to 03:00 UT on 5 August 2010. All parameters are expressed in units of $10^{-10}$ dyn $\mathrm{cm}^{-2}$.

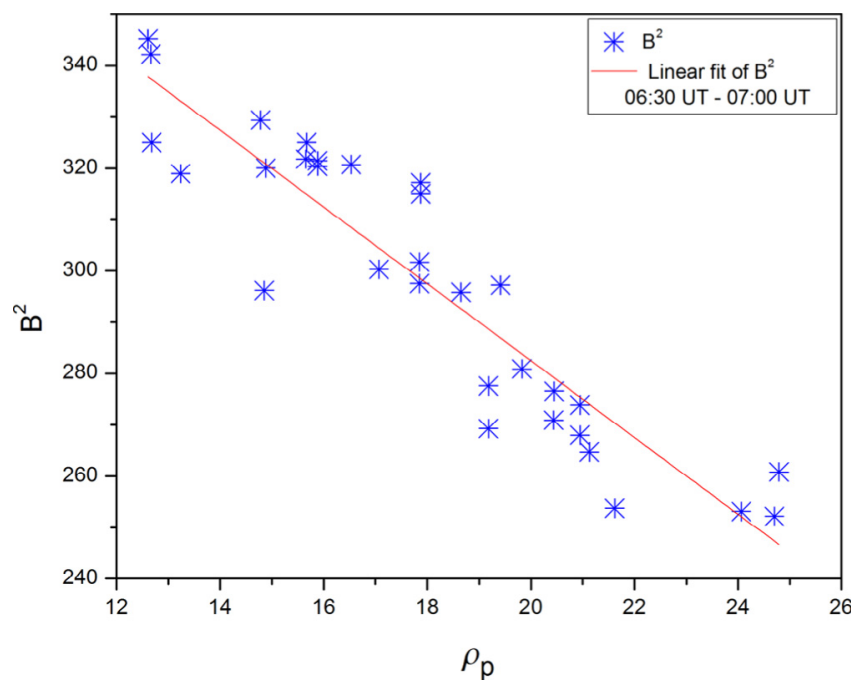

Fig. 19. Plot between square of magnetic field strength versus proton density for time interval 06:30-07:00 UT on 4 August 2010.

computed by Wind and ACE data. The features we are interested in are appeared in magnetic clouds (MC1 and MC2) at 04:20 UT and 10:32 UT respectively on 4 August. The microstructures can be seen over a scale of 10-60 min.

Square of magnitude of magnetic field strength $\left(B^{2}\right)$ is plotted with proton density and found to have a negative slope during intervals of PBS. Figure 19 shows plots of $B^{2}$ versus proton density for interval 06:30-07:30 UT on 4 August 2010, with regression coefficient $(R=-0.92)$.

\subsubsection{Compositional signatures}

Presence of filamentary plasma as low charge state species is observed in datasets from SWICS/ACE. Plots of hourly averaged charge state distribution from 4 to 7 August 2010 in Figure 20 show low charge states of carbon, oxygen and iron with high ion density during the intervals in magnetic clouds, believed to 


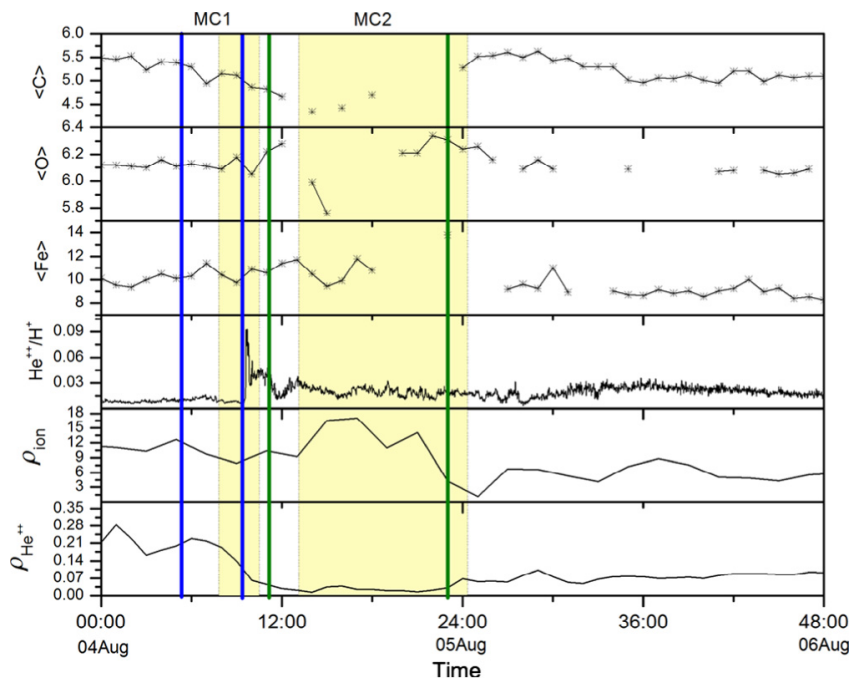

Fig. 20. Compositional signatures of filament plasma from 1-h averaged ACE/SWICS data from 4 to 6 August 2010. Average charge distribution (carbon, oxygen, iron), 1-min averaged alpha to proton ratio from $3 \mathrm{DP} /$ Wind, ion density and helium density are plotted from top to bottom.

be associated with filament eruption. The filamentary plasma arrived as a part of the magnetic cloud (MC1) from 06:00 09:00 UT on 4 August and also in rear of second magnetic cloud (MC2) from 13:00 UT on 4 August to 03:00 UT on 5 August. In the regions with elevated ion, electron and proton densities, the charge state of carbon gets as low as 4.3 while the oxygen and iron charge states decreased to 5.7 and 8.2 respectively in magnetic clouds (MC1 and $\mathrm{MC} 2$ ). $\mathrm{He}^{++} / \mathrm{H}^{+}$ratio attains a peak in $\mathrm{MC1}$ with magnitude 0.09 . The $\mathrm{He}^{++} / \mathrm{H}^{+}$ratio in solar wind also shows solar cycle dependencies (Ogilvie \& Hirshberg 1974; Feldman et al. 1978; Neugebauer 1981; Ogilvie et al. 1989), which leads to a decreased proton flux at $2.5 R_{\odot}$ during solar minimum. This reduction of the proton flux decreases the efficiency of the Coulomb drag and reduces the $\mathrm{He}^{++} / \mathrm{H}^{+}$ratio in solar wind (Aellig et al. 2001). Here, the average $\mathrm{He}^{++} / \mathrm{H}^{+}$ratio in ambient solar wind is $0.01-0.02$. The region in MC2 shows an elevated plateau of proton and electron densities, implying a diffused plasma cloud of filament plasma.

Further, the $\mathrm{He}^{+}$and $\mathrm{He}^{++}$count rates from CELIAS/STOF show (Fig. 21) a sharp increase on 3 August with high $\mathrm{He}^{+} /$ $\mathrm{He}^{++}$ratio. Instrument has detected two counts for $\mathrm{He}^{+}$ion while six counts for $\mathrm{He}^{++}$ions and a $\mathrm{He}^{+} / \mathrm{He}^{++}$ratio of 3 . It is here important to mention that the sensitivity of the STOF instrument on CELIAS/SOHO has degraded significantly since its launch. In this case, the count statistics is poor as compared to November 2003 event (M. Hilchenbach, priv. comm.).

The ratio of charge states (Fig. 22) of carbon and oxygen ions was taken by SWICS/ACE with data gaps in MC2 region. Thus, the freeze-in properties are only determined for $\mathrm{MC1}$ where the $\mathrm{C}^{6} / \mathrm{C}^{5}$ ratio drops to 0.15 in filament plasma region. Similar trend is observed with oxygen charge state ratios which shows a dip to 0.1 in filament region.

The $\mathrm{Fe} / \mathrm{O}$ ratio peaked at rear boundary of $\mathrm{MCl}$ with a value around 4.2 which is about $\approx 5$ times higher than that in ambient plasma (0.08). For a carbon charge state ratio of 0.15 in filament plasma region, the computed freeze-in temperature is found to be $6.02 \times 10^{5} \mathrm{~K}$ while for oxygen charge state ratio of 0.18 , the computed temperature is $5.74 \times 10^{5} \mathrm{~K}$.

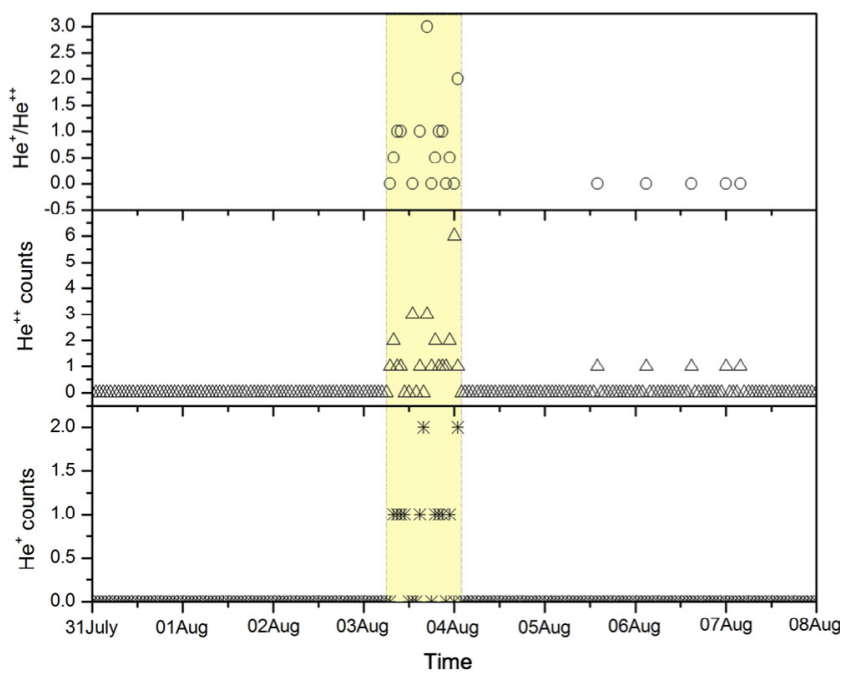

Fig. 21. Helium count rates and ratio from CELIAS/STOF onboard SOHO from 31 July to 8 August 2010. Filamentary plasma is estimated to arrive on 3-4 August. Count rates in 100-300 keV energy range are marked as star for $\mathrm{He}^{+}$counts, triangle for $\mathrm{He}^{++}$ counts and circles for the $\mathrm{He}^{+} / \mathrm{He}^{++}$ratio (courtesy: Dr. Martin Hilchenbach).

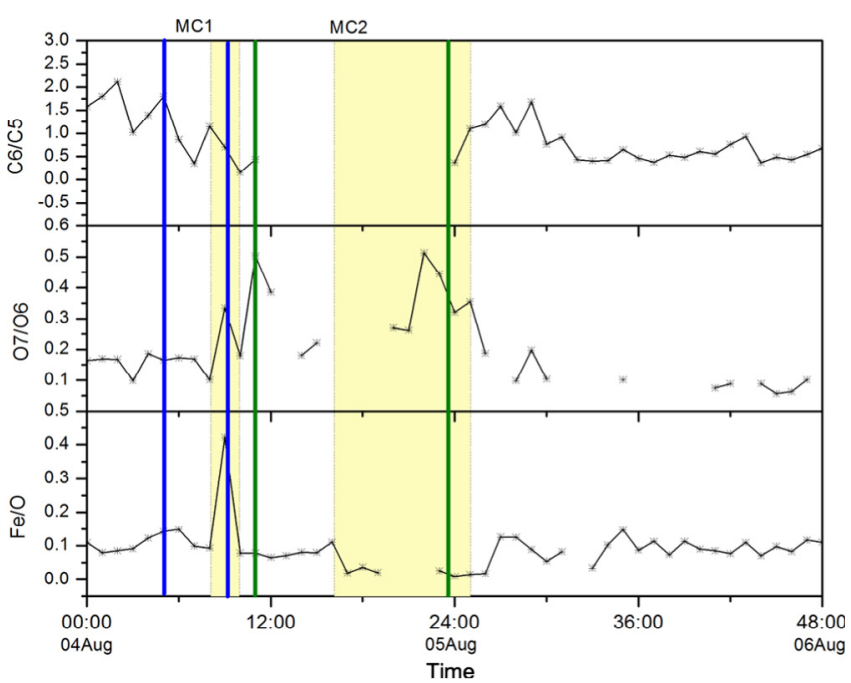

Fig. 22. Charge state ratios of carbon, oxygen ions and $\mathrm{Fe} / \mathrm{O}$ ratio from 4 to 6 August 2010 obtained from SWICS/ACE $-1 \mathrm{~h}$ resolution data.

\section{Discussion}

In this paper, we detected the presence of filament plasma in two ICMEs observed during different phases of solar cycle. Magnetic and plasma parameters were used along with compositional signatures to investigate the filament ejecta at $1 \mathrm{AU}$. We studied magnetic field signatures to identify magnetic clouds and flux rope configuration therein. The presence of filament plasma over polarity inversion line is consistent with filament models from Martens \& Kuin (1989) and Martens \& Zwaan (2001). Plasma properties for filament plasma such as low temperature, high proton and electron densities coincided with pressure balanced regions in magnetic clouds. Compositional signatures such as depressed ion charge states and high ion and helium densities and presence of $\mathrm{He}^{+}$ions were also observed in filament plasma region. Studied ICMEs appear to 
R. Sharma and N. Srivastava: Solar filament plasma identification in ICMEs

Table 1. Summary of plasma and magnetic parameters associated with studied ICMEs. Y = yes, '-' = not observed.

\begin{tabular}{|c|c|c|}
\hline Characteristics & 20 November 2003 ICME & 4 August 2010 ICME \\
\hline$T_{\mathrm{p}}, T_{\mathrm{e}}$, decrease & $\mathrm{Y}$ & $\mathrm{Y}$ \\
\hline Anisotropy $T_{\mathrm{p}}$ & $\geq 1$ & $\geq 1$ \\
\hline Anisotropy $T_{\mathrm{e}}$ & $\geq 1$ & $\leq 1$ \\
\hline $\mathrm{B}$ increase & $\overline{\mathrm{Y}}$ & $\overline{\mathrm{Y}}$ \\
\hline Proton, electron, ion and helium density increase & Y & Y \\
\hline $\mathrm{He}^{++} / \mathrm{H}^{+}$and $\mathrm{He}^{+} / \mathrm{He}^{++}$abundance ratio increase & Y & $\mathrm{Y}$ \\
\hline $\mathrm{He}^{+}$presence & Y & $\mathrm{Y}$ \\
\hline Decrease in carbon and oxygen charge states & Y & $\mathrm{Y}$ \\
\hline Decrease in iron charge states & - & Y \\
\hline Presence of trailing plasma outside $\mathrm{MC}$ & Y & Y \\
\hline Filament plasma position in $\mathrm{MC}$ & Behind $\mathrm{MC}$ axis & Behind $\mathrm{MC}$ axis \\
\hline Filament plasma location in $\mathrm{MC}$ & Rear & Rear \\
\hline Depression in RMS deviations of $V_{\mathrm{th}}, V, T_{\mathrm{p}}$ and $B$ & $\mathrm{Y}$ & Y \\
\hline Radial extension of filament plasma & $0.07 \mathrm{AU}$ & $0.19 \mathrm{AU}$ \\
\hline Ratio of radial extension of filament plasma and MC & 0.36 & 0.79 \\
\hline Decrease in thermal velocities & $\mathrm{Y}$ & $\mathrm{Y}$ \\
\hline Freezed-in temperature depressions & $\mathrm{Y}$ & $\mathrm{Y}$ \\
\hline
\end{tabular}

have a mixture of cold and hot plasma, which were also reported in other in situ plasma studies by Gloeckler et al. (1998) and in combination with presence of $\mathrm{He}^{+}$ions by Gosling et al. (1980). The rarity of finding cold, low charge state ions at $1 \mathrm{AU}$ as filament plasma suggests partial ionization of elements during transit through corona and development of magnetically confined plasmoids of mixed charge states (Neukomm \& Bochsler 1996; Skoug et al. 1999). It is thus possible that filament material is regularly observed in ICMEs at $1 \mathrm{AU}$, but is not recognized as such because it is no longer distinguished as ionizationally cold (Skoug et al. 1999). Signatures were also seen as depressions in root mean square deviations in ICMEs. Magnetic and plasma parameters such as magnetic field strength $(B)$, thermal $\left(V_{\text {th }}\right)$ and bulk $(V)$ velocities and temperature showed lower magnitude of RMS deviations in filament plasma regions. The observed signatures can be summarized in Table 1.

Previously reported cases of filament detection in ICMEs by Burlaga et al. (1998), Gopalswamy et al. (1998), Yao et al. (2010) and Lepri \& Zurbuchen (2010) were all focused on identifying and reporting the most ideal cases of filament plasma in ICMEs. Despite the fact that most of the solar eruptions are associated with filaments, only a few of them have been reported to contain cold material with low charge state species. This could possibly be due to location of the spacecraft, single point observations and/or due to heating of filamentary material as a result of a physical mechanism with solar (Filippov \& Koutchmy 2002) or in situ origins (Burlaga \& Behannon 1987; Burlaga 1995; Smith et al. 2001). The data analysis carried out in this study is limited to a single point observation, whereas ICMEs are three-dimensional structures that can be truly understood only through multipoint observations. The magnetic, plasma and compositional parameters were found to be in the same range despite the fact that the two ICMEs originated from different source regions at different phases of solar cycle. Use of different instruments, data analysis techniques and selection criteria also influences the identification of prominence plasma in ICMEs.

The in situ observations of ICMEs are a consequence of the physical mechanisms of their acceleration and injection into interplanetary medium and transportation along interplanetary magnetic field. In this paper, we have adopted the most practical approach to examine as many signatures as possible and identify the filament plasma based on grouping of several signatures within a certain region which can be assumed to be associated with filamentary plasma. The selected region might have distinct boundaries in plasma, magnetic and other signatures since they arise from different physical mechanisms and travel through different ambient medium at different phases of solar cycle.

Acknowledgements. We would like to thank P. Venkatakrishnan, Bernd Inhester and Shou Yao for useful discussions on the subject. The solar wind data from Wind and ACE spacecraft were obtained from NASA CDAWeb (http://cdaweb.gsfc.nasa.gov). We are grateful to Ruth Skoug for providing plasma data from SWEPAM/ ACE, Martin Hilchenbach and Harald Kucharek for providing helium ion data from CELIAS/STOF instrument. The work presented here by R.S. was carried out during his Student Project Traineeship at Udaipur Solar Observatory/Physical Research Laboratory. R.S. also acknowledges financial support from the organizers of SPACE CLIMATE-4 conference to attend the meeting at Goa, during 1621 January 2011 . The work by N.S. partially contributes to the research for European Union Seventh Framework Programme (FP7/ 2007-2013) for the Coronal Mass Ejections and Solar Energetic Particles (COMESEP) project under Grant Agreement No. 263252.

\section{References}

Aellig, M.R., A.J. Lazarus, and J.T. Steinberg, The solar wind helium abundance: variation with wind speed and the solar cycle, Geophys. Res. Lett., 28 (14), 2767-2770, 2001.

Alexander, D., I.G. Richardson, and T.H. Zurbuchen, A brief history of CME science, Space Sci. Rev., 123, 3-11, 2006.

Bame, S.J., J.R. Asbridge, W.C. Feldman, M.D. Montgomery, and P.D. Kearney, Solar wind heavy ion abundances, Sol. Phys., 43, 463, 1975

Bame, S.J., J.R. Asbridge, W.C. Feldman, E.E. Fenimore, and J.T. Gosling, Solar wind heavy ions from flare heated coronal plasma, Sol. Phys., 62, 179-201, 1979.

Bothmer, V., and R. Schwenn, Eruptive prominences as sources of magnetic clouds in the solar wind, Space Sci. Rev., 70, 215, 1994.

Brueckner, G.E., R.A. Howard, M.J. Koomen, C.M. Korendyke, D.J. Michels, et al., The large angle spectroscopic coronagraph (LASCO), Sol. Phys., 162, 357, 1995.

Burlaga, L.F., Micro-scale structures in the interplanetary medium, Sol. Phys., 4, 67, 1968. 
Burlaga, L.F., Interplanetary Magnetohydrodynamics, Oxford University Press, Oxford, ISBN-0-19-508472-1, 1995.

Burlaga, L.F., and K.W. Behannon, Compound streams, magnetic clouds and major geomagnetic storms, J. Geophys. Res., 92, 5725, 1987.

Burlaga, L.F., and K.W. Ogilvie, Magnetic and thermal pressures in the solar wind, Sol. Phys., 15, 61-71, 1970.

Burlaga, L.F., L.W. Klein, N.R. Sheeley Jr., D.J. Michels, R.A. Howard, M.J. Koomen, R. Schwenn, and H. Rosenbauer, A magnetic cloud and a coronal mass ejection, Geophys. Res. Lett., 9, 317, 1982.

Burlaga, L.F., J.D. Scudder, L.W. Klein, and P.A. Isenberg, Pressurebalanced structures between $1 \mathrm{AU}$ and $24 \mathrm{AU}$ and their implications for solar wind electrons and interstellar pickup ions, J. Geophys. Res., 95, 2229, 1990.

Burlaga, L., R. Fitzenreiter, R. Lepping, K. Ogilvie, A. Szabo, et al., A magnetic cloud containing prominence material: January 1997, J. Geophys. Res., 103 (A1), 277-285, 1998.

Chandra, R., E. Pariat, B. Schmieder, C.H. Mandrini, and W. Uddin, How can a negative magnetic helicity active region generate a positive helicity magnetic cloud? Sol. Phys., 261, 127-148, 2010.

Chandra, R., B. Schmieder, C. Mandrini, P. Demoulin, E. Pariat, et al., Homologous flares and magnetic field topology in active region NOAA 10501 on 20 November 2003, Sol. Phys., 269, 83104, DOI: 10.1007/s11207-010-9670-9, 2011.

Crooker, N.U., and T.S. Horbury, Solar imprint on ICMEs, their magnetic connectivity and heliospheric evolution, Space Sci. Rev., 123, 93-109, 2006.

Feldman, W.C., J.R. Asbridge, S.J. Bame, and J.T. Gosling, Longterm variations of selected solar wind properties: Imp 6, 7, and 8 results, J. Geophys. Res., 83, 2177-2189, 1978.

Feldman, W.C., B.L. Barraclough, and J.L. Phillips, Constraints on high-speed solar wind structure near its coronal base: a Ulysses perspective, $A \& A, \mathbf{3 1 6}, 355,1996$.

Ferraro, V.C.A., and C. Plumpton, An Introduction to Magneto-Fluid Dynamics, Clarendon Press, Oxford, 1966.

Filippov, B., and S. Koutchmy, About the prominence heating mechanisms during its eruptive phase, Sol. Phys., 208, 283-295, 2002.

Forsyth, R.J., V. Bothmer, C. Cid, N.U. Crooker, T.S. Horbury, et al., ICMEs in the inner heliosphere: origin, evolution and propagation effects, Space Sci. Rev., 123, 383-416, 2006.

Galvin, A.B., Minor ion composition in CME-related solar wind, In Coronal Mass Ejections, eds. N., Crooker, J.A. Joselyn, and J. Feynmann, AGU, Washington, 253, 1997.

Gary, S.P., L. Yin, D. Winske, J.T. Steinberg, and R.M. Skoug, Solar wind ion scattering by Alfven-cyclotron fluctuations: ion temperature anisotropies versus relative alpha particle densities, New J. Phys., 8, 17, 2006.

Geiss, J., G. Gloeckler, R. von Steiger, H. Balsiger, L.A. Fisk, et al., The southern high-speed stream - results from the SWICS instrument on Ulysses, Science, 268, 1033, 1995.

Gloeckler, G., J. Cain, F.M. Ipavich, E.O. Tums, P. Bedini, et al., Investigation of the composition of solar and interstellar matter using solar wind and pickup ion measurements with SWICS and SWIMS on the ACE spacecraft, Space Sci. Rev., 86, 497, 1998.

Gonzalez, W.D., J.A. Joselyn, Y. Kamide, H.W. Kroehl, G. Rostoker, et al., What is a geomagnetic storm? J. Geophy. Res., 99 A4, 5771-5792, 1994.

Gopalswamy, N., Properties of interplanetary coronal mass ejections, Space Sci. Rev., 124, 145-168, DOI: 10.1007/s11214-006-9102-1, 2006.

Gopalswamy, N., Y. Hanaoka, T. Kosugi, R.P. Lepping, J.T. Steinberg, et al., On the relationship between coronal mass ejections and magnetic clouds, Geophys. Res. Lett., 25 (14), 2485-2488, 1998.

Gopalswamy, N., S. Yashiro, G. Michalek, H. Xie, R.P. Lepping, and R.A. Howard, Solar source of the largest geomagnetic storm of cycle 23, Geophys. Res. Lett., 32, L12S09,

DOI: 10.1029/2004GL021639, 2005.
Gosling, J.T., V. Pizzo, and S.J. Bame, Anomalously low proton temperatures in the solar wind following interplanetary shock waves - evidence for magnetic bottles? J. Geophys. Res., 78, 2001, 1973.

Gosling, J.T., J.R. Asbridge, S.J. Bame, and W.C. Feldman, Observations of large fluxes of $\mathrm{He}^{+}$in the solar wind following an interplanetary shock, J. Geophys. Res., 85, 3431, 1980.

Harrison, R.A, J.A. Davies, C. Möstl, Y. Liu, M. Temmer, et al., An analysis of the origin and propagation of the multiple coronal mass ejection of 2010 August 1, Astrophys. J., 750, 45, DOI: 10.1088/0004-637X/750/1/45, 2012.

Hovestadt, D., M. Hilchenbach, A. Bürgi, B. Klecker, P. Laeverenz, et al., CELIAS - Charge, Element and Isotope Analysis System for SOHO, Sol. Phys.,162, 441, 1995.

Howard, R.A., D.J. Michels, N.R. Sheeley Jr., and M.J. Koomen, The observation of a coronal transient directed at Earth, Astrophys. J., 263, 1982.

Howard, R.A., J.D. Moses, A. Vourlidas, J.S. Newmark, D.G. Socker, et al., Sun earth connection coronal and heliospheric investigation (SECCHI), Space Sci. Rev., 136, 67-115, 2008.

$\mathrm{Hu}$, Q., and B.U.Ö. Sonnerup, Reconstruction of magnetic clouds in the solar wind: orientations and configurations, J. Geophys. Res., 107 (A7), 1142, DOI: 10.1029/2001JA000293, 2002.

Hudson, H.S., J.L. Bougeret, and J. Burkepile, Coronal mass ejections: overview of observations, Space Sci. Rev., 123, 13, 2006.

Hundhausen, A.J., Coronal expansion and solar wind, SpringerVerlag, New York, 1972.

Hundhausen, A.J., The origin and propagation of coronal mass ejections, in Solar Wind Six, eds. V.J., Pizzo, T.E. Holzer, and D.G. Sime, Proc, Natl. Cent. for Atmos. Res., Boulder, Colo, p. 181, Tech. Note, $306,1988$.

Hundhausen, A.J., H.E. Gilbert, and S.J. Bame, Ionization state of the interplanetary plasma, J. Geophys. Res., 73, 5485, 1968 a.

Hundhausen, A.J., H.E. Gilbert, and S.J. Bame, The state of ionization of oxygen in the solar wind, Astrophys. J., 152, 1968 b.

Klein, L.W., and L.F. Burlaga, Interplanetary magnetic cloud at 1 AU, J. Geophys. Res., 87, 613, 1982.

Kumar, P., P.K. Manoharan, and W. Uddin, Multiwavelength study on solar and interplanetary origins of the strongest geomagnetic storm of solar cycle 23, Sol. Phys., 271 (1-2), 149-167, 2011.

Lemen, J.R., A.M. Title, C. Akin, J.F. Drake, D.W. Duncan, et al., Atmospheric imaging assembly (AIA) on the solar dynamics observatory (SDO), Sol. Phys., 275, 17-40,

DOI: 10.1007/s11207-011-9776-8, 2011.

Lepping, R.P., M.H. Acuna, L.F. Burlaga, W.M. Farrell, J.A. Slavin, et al., The WIND magnetic field investigation, Space Sci. Rev., 207, 1995.

Lepri, S.T., and T.H. Zurbuchen, Direct observational evidence of filament material within interplanetary coronal mass ejections, Astrophys. J. Lett., 723, 22-27, DOI: 10.1088/2041-8205/723/1/L22, 2010.

Lopez, R.E., Solar cycle invariance in solar wind proton temperature relationships, J. Geophys. Res., 92 (A10),

DOI: 10.1029/JA092iA10p11189, 1987.

Lopez, R.E., and J.W. Freeman, Solar wind proton temperaturevelocity relationship, J. Geophys. Res., 91, 1701-1705, 1986.

Marsch, E., and C.Y., Tu, Evidence for pitch angle diffusion of solar wind protons in resonance with cyclotron waves, J. Geophys. Res., 106, 8357, 2001.

Marsch, E., K.H. Mühlhäuser, R. Schwenn, H. Rosenbauer, W. Pilipp, and F.M. Neubauer, Solar wind protons: three dimensional velocity distributions and derived plasma parameters measured between 0.3 and $1 \mathrm{AU}, J$. Geophys. Res., 87 (A1), 52-72, 1982.

Marsch, E., X.Z. Ao, and C.Y. Tu, On the temperature anisotropy of the core part of the proton velocity distribution function in the solar wind, J. Geophys. Res., 109, A04102, DOI: 10.1029/2003JA010330, 2004.

Martens, P.C.H., and N.P.M. Kuin, A circuit model for filament eruptions and two ribbon flares, Sol. Phys., 122, 263-302, 1989. 
Martens, P.C., and C. Zwaan, Origin and evolution of filamentprominence systems, Astrophys. J., 558, 872-887, 2001.

Marubashi, K., Structure of interplanetary magnetic clouds and their solar origins, Adv. Space Res., 6 (6), 33, 1986.

McComas, D.J., S.J., Bame, P., Barker, W.C., Feldman, J.L., Phillips, P., Riley, and J.W., Griffee, Solar Wind Electron Proton Alpha Monitor (SWEPAM) for the Advanced Composition Explorer, Space Sci. Rev., 86, 563-612, 1998.

Möstl, C., C. Miklenic, C.J. Farrugia, M. Temmer, A. Veronig, A.B. Galvin, B. Vršnak, and H.K. Biernat, Two-spacecraft reconstruction of a magnetic cloud and comparison to its solar source, Ann. Geophys., 26, 3139-3152, 2008

Möstl, C., C.J. Farrugia, E.K.J. Kilpua, L. Jian, and Y. Liu, Multipoint shock and flux rope analysis of multiple interplanetary coronal mass ejections around 2010 August 1 in the inner heliosphere, Astrophys. J., 2012, in press.

Neugebauer, M., Observations of solar wind helium, Fund. Cosmic Phys., 7, 131, 1981

Neugebauer, M., B.E. Goldstein, D. Winterhalter, E.J. Smith, R.J. MacDowall, and S.P. Gary, Ion distributions in large magnetic holes in the fast solar wind, J. Geophys. Res., 106, 5635, 2001.

Neukomm, R.O., and P. Bochsler, Diagnostics of closed magnetic structures in the solar corona using charge states of helium and of minor ions, Astrophys. J., 465, 462, 1996.

Ogilvie, K.W., and J. Hirshberg, The solar cycle variation of the solar wind helium abundance, J. Geophys. Res., 79, 4595-4602, 1974.

Ogilvie, K.W., M.A. Coplan, and P. Bochsler, Solar wind observations with the ion composition instrument aboard the ISEE-3/ICE spacecraft, Sol. Phys., 124, 167-183, 1989.

Ogilvie, K.W., D.J. Chornay, R.J. Fritzenreiter, F. Hunsaker, J. Keller, et al., SWE, a comprehensive plasma instrument for the WIND spacecraft, Space Sci. Rev., 71, 55, 1995.

Owocki, S.P., and J.D. Scudder, The effect of a non-Maxwellian electron distribution on oxygen and iron ionization balances in the solar wind, Astrophys. J., 270, 758, 1983.

Pudovkin, M.I., S.A. Zaitseva, and E.E. Benevolenska, The structure and parameters of flare streams, J. Geophys. Res., 84 (A11), 6649-6652, DOI: 10.1029/JA084iA11p06649, 1979.

Richardson, I.G., and H.V. Cane, Regions of abnormally low proton temperature in the solar wind (1965-1991) and their association with ejecta, J. Geophys. Res., 100, 23397-23412, 1995.

Schmieder, B., P. D'emoulin, E. Pariat, T. Török, Molodij, et al., Actors of the main activity in large complex centres during the 23 solar cycle maximum, Adv. Space Res., 47, 2081-2091, DOI: 10.1016/j.asr.2011.02.001, 2011.

Schwenn, R., H. Rosenbauer, and K.H. Mühlhäuser, Singly ionized helium in the driver gas of an interplanetary shock wave, Geophys. Res. Lett., 7 (3), 201-204, 1980.

Schwenn, R., J.C. Raymond, D. Alexander, A. Ciaravella, N. Gopalswamy, et al., Coronal observations of CMEs: report of Working Group A, Space Sci. Rev., 123, 127-176, 2006.

Shull, J.M., and M. van Steenberg, The ionization equilibrium of astrophysically abundant elements, Astrophys. J. Suppl., 48, 95, 1982
Skoug, R.M., S.J. Bame, W.C. Feldman, J.T. Gosling, D.J. McComas, et al., A prolonged $\mathrm{He}^{+}$enhancement within a coronal mass ejection in the solar wind, Geophys. Res. Lett., 26 (2), 161164, DOI: 10.1029/1998GL900207, 1999.

Smith, C.W., J. L'Heureux, N.F. Ness, M.H. Acuna, L.F. Burlaga, and J. Scheifele, The ACE Magnetic Field Experiment, Space Sci. Rev., 86 (1-4), 613-632, 1998.

Smith, C.W., W.H. Matthaeus, G.P. Zank, N.F. Ness, S. Oughton, and J.D. Richardson, Heating of the low-latitude solar wind by dissipation of turbulent magnetic fluctuations, J. Geophys. Res., 106, 8253-8272, 2001.

Srivastava, N., S.K. Mathew, and R.E. Louis, Source region of the 18 November 2003 coronal mass ejection that led to the strongest magnetic storm of cycle 23, J. Geophys. Res., 114, A03107, 2009.

Temmer, M., B. Vrsnak, T. Rollett, B. Bein, and C.A. de Koning, Characteristics of the kinematics of a coronal mass ejection during the 2010 August 1 CME-CME interaction event, Astrophys. J., 749, 57, DOI: 10.1088/0004-637X/749/1/57, 2012.

Tu, C.Y., and E. Marsch, Anisotropy regulation and plateau formation through pitch angle diffusion of solar wind protons in resonance with cyclotron waves, J. Geophys. Res., 107, 1249 , DOI: 10.1029/2001JA000150, 2002.

von Steiger, R., R.F. Wimmer-Schweingruber, J. Geiss, and G. Gloeckler, Abundance variations in the solar wind, Adv. Space Res., 15 (7), 3-12, 1995.

Wang, Y., G. Zhou, P. Ye, S. Wang, and J. Wang, A study of the orientation of interplanetary magnetic clouds and solar filaments, Astrophys. J., 651, 1245-1255, 2006.

Wilson, R.M., and E. Hildner, Are interplanetary magnetic clouds manifestations of coronal transients at 1 AU? Sol. Phys., 91, 169, 1984.

Wilson, R.M., and E. Hildner, On the association of magnetic clouds with disappearing filaments, J. Geophys. Res., 91, 5867, 1986.

Yao, S., E. Marsch, C.Y. Tu, and R. Schwenn, Identification of prominence ejecta by the proton distribution function and magnetic fine structure in interplanetary coronal mass ejections in the inner heliosphere, J. Geophys. Res., 115, A05103, DOI: 10.1029/2009JA014914, 2010.

Yurchyshyn, V., Q. Hu, and V. Abramenko, Structure of magnetic fields in NOAA active regions 0486 and 0501 and in the associated interplanetary ejecta, Space Weather, 3, S08C02, 2005.

Zurbuchen, T.H., and I.G. Richardson, In-situ solar wind and magnetic field signatures of interplanetary coronal mass ejections, Space Sci. Rev., 123, 31-43, DOI: $10.1007 / \mathrm{s} 11214-006-9010-4,2006$

Zwickl, R.D., J.R. Asbridge, S.J. Bame, W.C. Feldman, J.T. Gosling, and E.J. Smith, Plasma properties of driver gas following interplanetary shocks observed by ISEE3, in Solar Wind Five, ed. M., Neugebauer, NASA Conf. Publ., CP2280, 711-717, 1983. 